\title{
Emergent zooplankton in Moreton Bay, Queensland, Australia: seasonal, lunar, and diel patterns in emergence and distribution with respect to substrata
}

\author{
C. A. Jacoby* , J. G. Greenwood \\ Department of Zoology, University of Queensland, St. Lucia, Brisbane, Queensland 4067, Australia
}

\begin{abstract}
Forty-three putative taxa were common in emergence trap, re-entry trap, and net tow samples taken in Moreton Báy, Queensland. Australia between 20 January and 14 February and between 14 July and 23 August 1985. Twenty-nine taxa were composed of adults with 17 groups classified as demersal zooplankters. The remaining 14 taxa consisted of early life history stages. Statistically significant factorial variations in numbers of zooplankters captured were found between seasons ( 2 to 500), among lunar periods ( 2 to 150), and among substrata (2 to 200). Summer samples yielded greater numbers of 25 of the 32 taxa composed of adults and 10 of the 14 larval groups. Ten of the 17 demersal taxa, 5 other groups of adults, and 5 larval forms emerged in greater numbers during either lunar quarter or the new moon. Significant emergence during full moons was observed for 9 of the 14 larval taxa, 5 demersal groups, and 5 other adult taxa. Forty-one out of the 43 taxa emerged in statistically greater numbers from 1 of the 2 more complex substrata, coral or seagrass, as compared to mud or coral rubble. Five different temporal emergence patterns were displayed by 34 taxa with most emergence occurring at night.
\end{abstract}

\section{INTRODUCTION}

Studies of zooplankton that associate with the substratum during the day and enter the water column at night have been centered on coral reefs. These investigations often involved the use of emergence or re-entry traps to capture migrating zooplankters. Numbers of animals captured varied with seasons, lunar periods, substratum types, and time of day (Alldredge \& King 1977, Porter \& Porter 1977, Porter et al. 1977, Feeley et al. 1979, Hobson \& Chess 1979, McWilliam et al. 1981, Walter et al. 1981, Ohlhorst 1982, Jacoby \& Greenwood 1988). Further use of these methods has identified noctural migrations of zooplankters from subtidal sand flats (Alldredge \& King 1980, 1985, Youngbluth 1982a, Stretch 1985), seagrass beds (Robichaux et al. 1981, Alldredge 1985, Bell et al. 1986, Hicks 1986, Walters \& Bell 1986), kelp beds (Hammer \& Zimmerman 1979 ,

\footnotetext{
- Present address: University of Auckland, Leigh Marine Laboratory, RD 1, Leigh, New Zealand
}

Hammer 1981), and a variety of substrata in a temperate estuary (Thomas \& Jelley 1971).

Demersal zooplankton, i.e. animals that regularly migrate into the water column at night from a daytime position in, on, or near the substratum, initially became of interest as a part of the trophic webs of coral reefs (Johnson 1949, Emery 1968, Hamner \& Carleton 1979). Members of this assemblage currently are considered an important food source for invertebrates and fishes in a variety of habitats (Porter 1974, Hobson \& Chess 1976 , 1978, Robertson \& Howard 1978, Nelson 1979, Alheit 1981, Alheit \& Scheibel 1982, Choat 1982, de Morais \& Bodiou 1984, Leber 1985). Estuaries represent nursery grounds for many fishes and invertebrates (McHugh 1967), therefore, demersal zooplankton in estuaries should represent a critical link to higher trophic levels.

This study was designed to examine behavior and distribution of demersal zooplankton in Moreton Bay, a subtropical estuary on the east coast of Australia. Reentry traps, net tows, and 2 types of emergence traps were used to characterize the zooplanktonic community. Analyses focused on variations in numbers of zoo- 
plankters captured from different substrata during different seasons, lunar periods, and times of day.

\section{MATERIALS AND METHODS}

General sampling procedure. Sampling was conducted at 2 locations in Moreton Bay $\left(27^{\circ} 20^{\prime} \mathrm{S} ; 153^{\circ} 15^{\prime}\right.$ E) southeast of Brisbane, Queensland, Australia. One locale was off Polka Point on the west side of North Stradbroke Island in 0.7 to $2.5 \mathrm{~m}$ of water with muddy sand (mud) or seagrass (grass) substratum. The second location had coral patches (coral) and a coral rubble (rubble) substratum in 0.7 to $3.5 \mathrm{~m}$ of water and was located along the east side of Goat Island ca $2.5 \mathrm{~km}$ southwest of Polka Point. Because the locations were subject to similar hydrological conditions, samples were attributed to 1 of the 4 substrata. It was recognized that other site-specific phenomena may have influenced the numbers of zooplankters captured.

Samples were taken between 20 January and 14 February and between 14 July and 23 August 1985. These periods represented the extremes in hydrological conditions and daylength. Salinity in Moreton Bay typically ranges from $34 \%$ in summer to $28 \%$ in winter (Newell 1971). A summer to winter decrease in water temperature from 25 to $13^{\circ} \mathrm{C}$ was recorded at both sites. Daylength was $13.5 \mathrm{~h}$ in summer and $10.5 \mathrm{~h}$ in winter. Comparisons were made between these 2 seasonal extremes although it was acknowledged that factors specific to the year 1985 may have altered the numbers of zooplankters captured.

Three types of sampling were used to characterize the distributions and behavior of zooplankton. A plankton net was towed at the surface during daytime and night-time to estimate the number and diversity of zooplankters reaching the upper $0.5 \mathrm{~m}$ of the water column. Re-entry traps were used to determine the abundance and diversity of zooplankters near the bottom during the day. Zooplankters migrating from near the bottom into the water column were captured in 2 types of emergence traps.

Net tows. A $200 \mu \mathrm{m}$ mesh plankton net with a $40 \mathrm{~cm}$ mouth was towed within $0.5 \mathrm{~m}$ of the surface during new moons. Night tows were made at 20:00 h on 24 January and at 19:30 on 19 July. Daytime samples were taken at 08:00 on 25 January and at 13:30 h on 19 July. A General Oceanics 2030 flowmeter attached to the net provided data needed to calculate the volumes of water filtered during the 5 min tows. All plankton net tows were made at the Polka Point site in ca $1.5 \mathrm{~m}$ of water These and all other samples were concentrated on a 100 um mesh sieve and preserved in $4 \%$ formaldehyde in seawater.

Re-entry traps. These consisted of $19.5 \times 26 \mathrm{~cm}(0.051$ $\mathrm{m}^{2}$ ) plastic trays that were $8.5 \mathrm{~cm}$ deep and could be sealed (Jacoby \& Greenwood 1988). The trays contained 5 to $7 \mathrm{~cm}$ of muddy sand from the Polka Point site. The sediment had been rinsed 10 times with freshwater to remove zooplankters and air-dried prior to deployment. Six traps were filled with filtered seawater, covered, and anchored on seagrass at the Polka Point site at 17:00 h on 11 February (last lunar quarter) and at $11: 30$ h on 15 July (new moon). All 6 trays were opened to permit colonization. Two traps were covered and retrieved between 08:30 and 09:00 h after each of 3 consecutive nights. A maximum of $1500 \mathrm{ml}$ of non-interstitial water was collected in each trap. Zooplankters were rinsed from the sediment with freshwater 5 times onto a $100 \mu \mathrm{m}$ sieve and then preserved. Trays were located on grass to ensure that colonizers had to move from the adjacent mud substratum to locate the available space. All comparisons were limited to samples taken from mud.

Net emergence traps. Four emergence traps made of $100 \mu \mathrm{m}$ mesh (net traps; Jacoby \& Greenwood 1988) were placed over $1 \mathrm{~m}^{2}$ patches of each substratum at each site for 3 nights during new moon periods in summer and winter. A total of 8 net traps were deployed over grass and mud substrata at Polka Point on 20, 24 . and 25 January and on 16, 17, and 19 July. Four net traps were set above coral and 4 traps were placed on rubble at Goat Island on 21, 22, and 23 January and on 14, 15, and 18 July. Coral and grass substrata were not composed of a single type of bottom. Coral formations had to be less than $1 \mathrm{~m}^{2}$ and $50 \mathrm{~cm}$ high to fit under the traps; therefore, net traps deployed over coral also covered some rubble. Seagrass at Polka Point grew from a muddy sand substratum similar to bare mud. Net traps were deployed between 14:30 and 15:30 h, and were retrieved between 08:30 and 09:30 h the next morning. The resulting $18 \mathrm{~h}$ sampling period was treated as 1 night in analysis of seasonal and substratum-related differences in emergence. Samples were concentrated and preserved as described previously

Rotary emergence traps. A second type of emergence trap, rotary emergence trap (RET; Youngbluth 1982a, b, Jacoby \& Greenwood 1988), was set over $1 \mathrm{~m}^{2}$ patches of grass and mud at the Polka Point site and over small coral formations off Goat Island. Once again, patches of coral and grass were not pure substrata. Two RETs were placed over each of the 3 types of bottom for 1 night during each lunar period in summer and winter Sampling began between $14: 30$ and $15: 30 \mathrm{~h}$ and ended between $11: 30$ and $12: 30$ h the following day. Fourteen separate samples of 90 min duration were taken during this $21 \mathrm{~h}$ period because the RETs have multiple cod ends which rotated into position to sample for a pre-set time interval. The 14 samples were treated as indicating time of first emergence to $\mathrm{ca} 70 \mathrm{~cm}$ (the point where zooplankters were trapped in the cod ends), and data from these samples were used in analyses of 
temporal patterns in emergence. Counts from all 14 samples were summed to yield numbers $\mathrm{m}^{-2}$ night $^{-1}$ which were analysed for seasonal, lunar, and substratum-related differences in emergence. Samples were sieved and concentrated as described above.

Analyses of samples and data. Zooplankters in all samples were identified and enumerated using a dissecting microscope. In general, full samples were counted, but extremely abundant taxa were counted in subsamples taken with a Stempel pipette. Only putative taxa taken with sufficient frequency to allow statistical analyses of their distributions were included.

Within each season, chi-square contingency tests (Zar 1974) were used to examine variation in the numbers of zooplankters captured among the 5 different types of samples and between daytime and night-time net tows. For summer data, mean numbers of zooplankters captured $\mathrm{m}^{-2}$ night ${ }^{-1}$ in re-entry traps during the last quarter were compared to mean numbers $\mathrm{m}^{-2}$ night ${ }^{-1}$ captured from mud by net traps and RETs during the new moon and to numbers $\mathrm{m}^{-3}$ taken in daytime and night-time net tows during the new moon. A similar comparison was made using winter data except that all 5 types of sampling were done during the new moon. Numbers of zooplankters $\mathrm{m}^{-3}$ taken in daytime and night-time net tows also were compared separately for each season.

Seasonal, exposure-related, lunar, substratumrelated, and diel variations in numbers of zooplankters captured by re-entry and emergence traps were examined using analyses of variance (ANOVAs) (SAS Institute Inc. 1985) and a posteriori Student-NewmanKeuls multiple comparison tests (S-N-K tests). Factors were considered fixed because a majority of all possible levels were represented in the analyses. Cochran's test was used to detect heterogeneity of variances prior to ANOVAs (Underwood 1981). Data typically were heteroscedastic with variances proportional to the means, so counts $(c)$ were $\log (c+1)$ transformed prior to analysis. Variances of some data sets remained heterogeneous; therefore, outlying data points were replaced with values equal to or proportional to the mean of the data set. This procedure reduced variance without altering the means. Degrees of freedom were removed from mean squares as appropriate (Underwood 1981). Significance levels were held at $p<0.05$ or $p<0.01$.

\section{RESULTS}

\section{General results}

Samples yielded 43 common, putative taxa. Taxonomic divisions varied from separate age and sex groups within a species to broad categories at the class level. Effort was concentrated on relatively common and numerous groups which were prospective demersal zooplankters; however results for all common taxa were included to illustrate contrasts in behavior and distribution

Numbers of all taxa varied greatly among replicate samples suggesting spatially and temporally patchy distributions. Outliers were replaced in most ANOVAs. This procedure may have increased Type I error : therefore, caution was used when considering results. In general, only one replacement was made for any taxon in an analysis. Furthermore, the total number of outliers replaced never exceeded $1 \%$ of the data, and the total number of non-zero outliers never exceeded $0.7 \%$ of the data. Variability was most extreme for adult copepods and copepodites in the calanoid genus Acartia (up to 900 times and 65 times respectively), polychaetes (up to 71 times), and caridean/anomuran larvae (up to 21 times).

\section{Comparisons among samplers}

Comparisons of the 5 types of sampling were restricted to results from mud. They revealed that the number of zooplankters captured within each season and taxon was not independent of trap type (Summer: chi-square $=83297.2, \mathrm{Df}=148, p<0.001$; Winter: chisquare $=17490.4, \mathrm{Df}=120, p<0.001)$. Within each season and taxon, the mean numbers of zooplankters captured $\mathrm{m}^{-2}$ or $\mathrm{m}^{-3}$ were summed across all 5 types of samples. This sum was used as the denominator for calculations of the percentage contribution by each type of sampling (Table 1). Twenty-five of the 38 taxa analysed were taken predominately in re-entry or emergence traps. A total of 13 taxa were reasonably common in net tows with 5 groups more numerous in night-time tows and 8 taxa occurring in approximately equal numbers in daytime and night-time net tows.

Four other taxa were too rare in the appropriate sample sets to be included in the analyses. Examination of all available data indicated that tunicate larvae and calanoid copepods in the genera Pseudocyclops and Ridgewayia were taken in greatest numbers in emergence traps. Isopods were captured mainly in emergence and re-entry traps. Some taxa absent from net tows, e.g. copepod nauplii, miscellaneous nauplii, and tunicate larvae, were too small to be sampled quantitatively by the $200 \mathrm{um}$ mesh net.

\section{Net tow data}

Numbers of zooplankters $\mathrm{m}^{-3}$ taken in net tows within each season and taxon were not independent of the time of the tow (Summer: chi-square $=256.2$, Df $=$ 
Table 1. Distribution of zooplankton captures by type of trap. Within a season, mean numbers of zooplankton $\mathrm{m}^{-2}$ or $\mathrm{m}^{-3}$ are not independent of taxon or type of trap as shown by chi-square contingency analyses $(p<0.05)$. Underlined percentages are at least $50 \%$ of the summed means within taxon and season and are considered significant. NA: summed means < 19; cop.: copepodites

\begin{tabular}{|c|c|c|c|c|c|c|}
\hline \multirow[t]{2}{*}{ Taxon/season } & \multirow{2}{*}{$\begin{array}{l}\text { Sum of } \\
\text { means }\end{array}$} & \multirow{2}{*}{$\begin{array}{l}\text { Re-entry } \\
\text { trap }\end{array}$} & \multicolumn{2}{|c|}{ Percent of summed means } & \multicolumn{2}{|c|}{ Tows } \\
\hline & & & $\mathrm{Nel}$ & RET & Night & Day \\
\hline \multicolumn{7}{|c|}{ Re-entry $>$ Emergence $>$ Tows } \\
\hline \multicolumn{7}{|c|}{ Pseudodiaptomus colefaxi cop. } \\
\hline Summer & 412 & $\frac{87.1}{607}$ & 0.8 & $\begin{array}{r}7.5 \\
28.6\end{array}$ & 2.7 & ${ }_{0}^{1.9}$ \\
\hline \multicolumn{6}{|l|}{ Peludium spp. } & 0 \\
\hline Summer & 50 & 72.0 & 8.0 & 20.0 & 0 & 0 \\
\hline Winter & 56 & 87.5 & 5.3 & 3.6 & 1.8 & 1.8 \\
\hline \multicolumn{7}{|l|}{ A.mphipods } \\
\hline Summer & 491 & 91.9 & 18 & 5.7 & 06 & 0 \\
\hline Winter & 753 & 85.0 & 3.3 & 10.9 & 0.8 & 0 \\
\hline Cumaceans & & & & & & \\
\hline Summer & 309 & 96.1 & 1.0 & 2.3 & 03 & 03 \\
\hline Winter & 583 & $\overline{98.1}$ & 03 & 14 & 0.2 & 0 \\
\hline Ostracods & & & & & & \\
\hline Summer & 1660 & 81.8 & 0.7 & 17.2 & 0.2 & 0.1 \\
\hline Winter & 230 & $\overline{91.0}$ & 65 & 1.7 & 0.4 & 04 \\
\hline Polychaetes & & & & & & \\
\hline Summer & 248 & 7900 & 65 & 12.1 & 1.5 & 0.8 \\
\hline Winter & 56 & 58.9 & 10.7 & $28 . \tilde{0}$ & 1.8 & 0 \\
\hline Gastropods & & & & & & \\
\hline Summer & 407 & 57.3 & 11.6 & 21.1 & 0 & 0 \\
\hline Winter & 69 & 75.4 & 10.2 & 11.6 & 1.4 & 1.4 \\
\hline Re-entry $=$ Emerge & & & & & & \\
\hline Pseudodiaptomu & & & & & & \\
\hline Summet & 285 & $\underline{80.4}$ & 9.1 & 7.7 & 1.8 & 1.0 \\
\hline Winter & 132 & $\overline{34.9}$ & 24.2 & 39.4 & 1.5 & 0 \\
\hline$P_{\text {seudodiaptomu }}$ & & & & & & \\
\hline Summer & 133 & 66.2 & 3.0 & 19.5 & 8.3 & 3.0 \\
\hline Winter & 135 & $\overline{41.5}$ & 5.2 & 50.4 & 2.2 & 0.7 \\
\hline Pseudodiaptomu & & & & & & \\
\hline Summer & 32 & 72.0 & 9.0 & 7.0 & 90 & 30 \\
\hline Winter & 47 & 27.7 & 40.4 & 29.8 & 2.1 & 0 \\
\hline Oithona spp. & & & & & & \\
\hline Summer & 6859 & 66.8 & 1.0 & 25.1 & 4.5 & 2.6 \\
\hline Winter & 861 & 36.2 & 5.5 & 51.6 & 5.6 & 1.1 \\
\hline Miscellaneous $\mathrm{h}$ & & & & & & \\
\hline Summer & 61463 & 92.5 & 0.6 & 6.7 & 01 & 0.1 \\
\hline Winter & 3592 & 47.7 & 6.7 & 44.9 & 06 & 0.1 \\
\hline Copepod nauplij & & & & & & \\
\hline Summer & 4759 & $\underline{53.3}$ & 2.2 & 44.5 & 0 & 0 \\
\hline Winter & 3940 & $\overline{77}$ & 8.3 & 84.0 & 0 & 0 \\
\hline Cirripede larvae & & & & & & \\
\hline Summer & 161 & 59.0 & 3.1 & 17.4 & 93 & 11.2 \\
\hline Winter & 111 & 11.7 & 28.8 & 54.1 & 4.5 & 0.9 \\
\hline Tanaids & & & & & & \\
\hline Summer & 28 & 36.0 & 110 & 430 & 70 & 30 \\
\hline Winter & 46 & $\underline{56.5}$ & 22 & 41.3 & 0 & 0 \\
\hline Polychaete larva & & & & & & \\
\hline Summer & 222 & 42.8 & 4.5 & 45.0 & 3.6 & 4.1 \\
\hline Winter & 201 & 16.4 & 10.5 & 70.6 & 2.0 & 05 \\
\hline Mysids & & & & & & \\
\hline Summer & 102 & 451 & 29 & 373 & 14.7 & 0 \\
\hline Winter & 94 & 383 & 32 & 468 & 11.7 & 0 \\
\hline Re-entry $=$ Night t & & & & & & \\
\hline Condeans & & & & & & \\
\hline Summer & 22 & 27.0 & 5.0 & 9.0 & 410 & 18.0 \\
\hline Winter & 9 & $\mathrm{NA}$ & & & & \\
\hline Emergence $>$ Re-e & & & & & & \\
\hline Stephos spp. & & & & & & \\
\hline Summer & 33 & 9.1 & 30 & $\frac{78.8}{579}$ & 30 & 6.1 \\
\hline $\begin{array}{l}\text { Winter } \\
\text { Paryocalons so }\end{array}$ & 19 & 0 & 421 & $\underline{579}$ & 0 & 0 \\
\hline $\begin{array}{l}\text { Parvocalanus sp } \\
\text { Summer }\end{array}$ & 165 & 1.8 & 4.8 & 758 & 9.7 & 7.9 \\
\hline Winter & 172 & 0.5 & 28 & 87.8 & 6.6 & 23 \\
\hline Parategastes $\mathrm{sp}$ & & & & & & \\
\hline Summer. & 442 & 2.9 & 61 & 90.3 & 05 & 0.2 \\
\hline Winter & 833 & 24.7 & 64 & 68.6 & 0.2 & $0 \mathrm{t}$ \\
\hline Miscellaneous al & & & & & & \\
\hline Summer & 28 & 0 & 7.0 & 930 & 0 & 0 \\
\hline Winter & 47 & 6.4 & 2.1 & 91.5 & 0 & 0 \\
\hline Zoeae & & & & & & \\
\hline Summer & 62 & 0 & 22.6 & 59.7 & 129 & 4.8 \\
\hline Winter & 86 & 0 & 1.2 & 55.8. & 4.6 & 38.4 \\
\hline
\end{tabular}


Table 1 (continued)

\begin{tabular}{|c|c|c|c|c|c|c|}
\hline \multirow[t]{2}{*}{ Taxon/seàson } & \multirow[t]{2}{*}{$\begin{array}{l}\text { Sum of } \\
\text { means }\end{array}$} & \multirow{2}{*}{$\begin{array}{l}\text { Re-entry } \\
\text { trap }\end{array}$} & \multicolumn{2}{|c|}{$\begin{array}{l}\text { Percent of summed means } \\
\text { Emergence traps }\end{array}$} & \multicolumn{2}{|c|}{ Tows } \\
\hline & & & Net & RET & Night & Day \\
\hline \multicolumn{7}{|c|}{ Emergence $>$ Re-entry $=$ Tows } \\
\hline \multicolumn{7}{|c|}{ Mets holothuriae } \\
\hline Summer & 66 & 15.2 & 3.0 & 75.8 & 1.5 & 4.5 \\
\hline \multirow{2}{*}{\multicolumn{7}{|c|}{ Caridean/anomuran larvae }} \\
\hline & & & & & & \\
\hline Summer & 91 & 0 & 12.1 & 76.9 & 77 & 3.3 \\
\hline Winter & 7 & NA & & & & \\
\hline \multicolumn{7}{|c|}{ Spadella cephaloptera } \\
\hline Summer & 34 & 8.8 & 3.0 & 88.2 & 0 & 0 \\
\hline Winter & 1 & NA & & & & \\
\hline \multicolumn{7}{|c|}{ Emergence $=$ Night tow $>$ Re-entry } \\
\hline \multicolumn{7}{|c|}{ Megalopa } \\
\hline Summer & 41 & 7.3 & 4.9 & 9.8 & 70.7 & 7.3 \\
\hline$W_{1 n t e r}$ & 26 & 0 & 3.8 & 69.2 & $\overline{25.9}$ & 0 \\
\hline \multicolumn{7}{|l|}{ Larvaceans } \\
\hline Summer & 170 & 5.9 & 5.9 & 21.2 & $\underline{51.7}$ & 15.3 \\
\hline Winter & 1579 & 0.2 & 1.3 & $\underline{97.5}$ & $\overline{0.9}$ & 0.1 \\
\hline \multicolumn{7}{|l|}{ Gastropod veligers } \\
\hline Summer & 4477 & 4.8 & 1.5 & 3.4 & 60.7 & 29.6 \\
\hline$W_{1} n t e r$ & 485 & 2.1 & 10.5 & 76.7 & $\overline{9.9}$ & 0.8 \\
\hline \multicolumn{7}{|l|}{ Bivalve veligers } \\
\hline Summer & 1123 & 0.5 & 27.3 & 39.4 & 28.5 & 4.3 \\
\hline Winter & 2262 & 0 & 41.4 & 58.1 & 0.4 & 0.1 \\
\hline \multicolumn{7}{|c|}{$\begin{array}{l}\text { All trap types approximately = } \\
\text { Acartia spp. adults }\end{array}$} \\
\hline $\begin{array}{l}\text { Acartia spp. adult } \\
\text { Summer }\end{array}$ & 315 & 20.7 & 5.4 & 10.1 & 29.5 & 34.3 \\
\hline $\begin{array}{l}\text { Summer } \\
\text { Winter }\end{array}$ & 1049 & 19.4 & 4.5 & 667 & 5.2 & 4.2 \\
\hline \multicolumn{7}{|l|}{ Acartla spp. cop. } \\
\hline Summer & 546 & 13.2 & 4.8 & 45.8 & 20.1 & 16.1 \\
\hline Winter & 2151 & 3.8 & 4.5 & 86.4 & 3.3 & 2.0 \\
\hline \multicolumn{7}{|l|}{ Calanoid cop. } \\
\hline Summer & 800 & 16.7 & 14 & 30.5 & 25.6 & 25.8 \\
\hline Winter & 1689 & 2.1 & 2.0 & $\underline{76.2}$ & 10.4 & 9.3 \\
\hline \multicolumn{7}{|c|}{ Miscellaneous cyclopoids } \\
\hline Summer & 307 & 24.4 & 2.3 & 164 & 26.1 & 30.3 \\
\hline Winter & 420 & 10.0 & 1.0 & 82.6 & 5.0 & 1.4 \\
\hline \multicolumn{7}{|c|}{ Miscellaneous calanolds } \\
\hline Summer & 498 & 17.1 & 2.0 & 8.0 & 44.0 & 28.9 \\
\hline Winter & 217 & 15.2 & 2.8 & 3.7 & 43.3 & 35.0 \\
\hline \multicolumn{7}{|c|}{ Pseudodiaptomus mertoni adults } \\
\hline Summer & 42 & 14.3 & 11.9 & 19.0 & 31.0 & 23.8 \\
\hline \multirow{2}{*}{\multicolumn{7}{|c|}{$\begin{array}{l}\text { Winter } \\
\text { Pseudodiaptomus mertonj cop. }\end{array}$}} \\
\hline & & & & & & \\
\hline Summer & 83 & 3.6 & 2.4 & 45.8 & 29.9 & 19.3 \\
\hline Winter & 3 & $\mathrm{NA}$ & & & & \\
\hline Sagitta spp. & & & & & & \\
\hline Summer & 196 & 11.8 & 1.0 & 16.8 & 32.1 & 38.3 \\
\hline Winter & 11 & $\mathrm{NA}$ & & & & \\
\hline
\end{tabular}

22, $p<0.001 ;$ Winter: chi-square $=114.7, \mathrm{Df}=12$, $p<0.001$ ). Comparisons of the proportions of zooplankters captured at night and during the day (Table 2) revealed that only zoeae were more common in any of the daytime net tows. In contrast, 10 groups of zooplankters were consistently more numerous in night-time net tows. A further 12 taxa were captured in approximately equal numbers in daytime and nighttime tows.

The remaining 19 taxa were too rare to be included in the analyses (total numbers taken less than $19 \mathrm{~m}^{-3}$ in all tows). Examination of the data for males and ovigerous females of the calanoid Pseudodiaptomus colefaxi, the harpacticoid Parategastes sp., amphipods, cumaceans, ostracods, tanaids, and polychaetes revealed that they were captured only in night tows. A harpacticoid, Metis holothuriae, was taken in both daytime and night-time tows. Calanoids in the genera Stephos,
Pseudocyclops, and Ridgewayia, as well as copepod nauplii, isopods, miscellaneous nauplii, the chaetognath Spadella cephaloptera, gastropods, and tunicate larvae, were extremely rare in all tows.

\section{Re-entry trap data}

ANOVAs applied to re-entry trap data indicated a significant effect due to season, night, and taxon (Cochran's value unadjusted $=0.06$, Df $=1,252$, $p<0.05$; Cochran's value adjusted $=0.03, p>0.05$, loss of $2 \mathrm{Df}_{;} F=1.94$, Df $=82,250, p<0.001$ ). S-N-K tests ( $\mathrm{SE}=0.36$ ) comparing abundances among the 42 taxa within each season and night indicated that miscellaneous harpacticoid copepods were the only zooplankters consistently captured in significantly greater numbers. 
Table 2. Distribution of zooplankton captures in surface net tows. Within a season, mean numbers captured $\mathrm{m}^{-3}$ are not independent of time of day as shown by chi-square contingency analyses $(p<0.05)$. Underlined percentages are over $60 \%$ of the grand mean within a season and are considered significant. NA: number $\mathrm{m}^{-3}<10$; cop: copepodites

\begin{tabular}{|c|c|c|c|c|c|c|}
\hline \multirow[t]{3}{*}{ Taxon } & \multicolumn{3}{|c|}{ Summer } & \multicolumn{3}{|c|}{ Winter } \\
\hline & \multirow[t]{2}{*}{ Total no. } & \multicolumn{2}{|c|}{$\%$ of mean } & \multirow[t]{2}{*}{ Total no. } & \multicolumn{2}{|c|}{$\%$ of mean } \\
\hline & & Night & Day & & Night & Dây \\
\hline \multicolumn{7}{|l|}{ Day > Night } \\
\hline Zoeae & 11 & $\underline{73}$ & 27 & 37 & 11 & $\underline{89}$ \\
\hline \multicolumn{7}{|l|}{ Night $>$ Day } \\
\hline Gastropod veligers & 4043 & $\underline{67}$ & 33 & 52 & $\underline{92}$ & 8 \\
\hline Oithona spp. & 484 & $\overline{63}$ & 37 & 58 & $\overline{83}$ & 17 \\
\hline Bivalve vehgers & 368 & $8 \overline{7}$ & 13 & 10 & 90 & 10 \\
\hline Larvaceans & 114 & 77 & 23 & 16 & 88 & 12 \\
\hline Miscellaneous harpacticoids & 100 & 54 & 36 & 25 & 88 & 12 \\
\hline Mysids & 15 & 100 & 0 & 11 & 100 & 0 \\
\hline Megalopa & 32 & 91 & 9 & 7 & $\overline{N A}$ & NA \\
\hline Pseudodiaptomus colefaxi \& 9 & 15 & 73 & 27 & 4 & NA & NA \\
\hline Candeans & 13 & $\overline{69}$ & 31 & 1 & NA & $\mathrm{NA}$ \\
\hline Caridear/anomuran larvae & 10 & $\underline{70}$ & 30 & 4 & NA & $\mathrm{NA}$ \\
\hline \multicolumn{7}{|l|}{ Day $=$ Night } \\
\hline Calanoid cop. & 411 & 50 & 50 & 332 & 53 & 47 \\
\hline Miscellaneous calanoids & 363 & 60 & 40 & 170 & 55 & 45 \\
\hline Acartia spp. adults & 201 & 46 & 54 & 99 & 56 & 44 \\
\hline Acartia spp. cop. & 198 & 56 & 44 & 114 & $\underline{62}$ & 38 \\
\hline Miscellaneous cyclopoids & 173 & 46 & 54 & 27 & $\overline{78}$ & 22 \\
\hline Parvocalanus sp. & 29 & 55 & 45 & 51 & $7 \underline{75}$ & 25 \\
\hline Sagitta spp & 138 & 46 & 54 & 9 & $\overline{N A}$ & NA \\
\hline$P_{\text {seudodiaptomus mertom adults }}$ & 23 & 56 & 44 & 2 & NA & NA \\
\hline Pseudadiaptornus mertoms cop. & 40 & 60 & 40 & 2 & NA & NA \\
\hline Cirripede larvae & 33 & 45 & 55 & 6 & NA & NA \\
\hline Pseudodiaptomus colefax cop. & 19 & 58 & 42 & 2 & $\mathrm{NA}$ & NA \\
\hline Polychaete larvae & 17 & 47 & 53 & 5 & $\mathrm{NA}$ & $\mathrm{NA}$ \\
\hline
\end{tabular}

Further multiple comparison tests revealed few significant differences among numbers of zooplankters captured $\mathrm{m}^{-2}$ among the 3 nights of exposure to colonization (Table 3). Differences were observed in 5 of the 42 taxa, but there was no consistent relation between changes in numbers captured and increased exposure.

Seasonal patterns in re-entry trap data were indicated by S-N-K tests for 19 out of 42 taxa (Table 4). Fifteen groups were taken in greater numbers during the summer. Acartia spp. adults and copepodites, as well as Parategastes sp., were more numerous in winter samples. Miscellaneous cyclopoid copepods, a broad taxon, did not display a consistent seasonal trend. Twenty-three taxa were captured in statistically equal numbers in both seasons.

\section{Net emergence trap data}

A significant interaction between season, substratum, and taxon was revealed by ANOVA applied to net emergence trap data (Cochran's value unadjusted $=0.04, \mathrm{Df}=11,336, p<0.05 ;$ Cochran's value adjusted $=0.009, p>0.05$, loss of $45 \mathrm{Df}, F=7.36, \mathrm{Df}=$ $123,3651, p<0.0001)$. S-N-K tests comparing numbers of zooplankters emerging $\mathrm{m}^{-2}$ night $^{-1}$ among the 42 taxa within each level of season and substratum ( $\mathrm{SE}=$ 0.09) revealed that cyclopoid copepods in the genus
Oithona, miscellaneous harpacticoid copepods, copepod nauplii, and bivalve veligers were the only groups regularly captured in statistically greater numbers.

Thirty-six of the 42 taxa exhibited significant seasonal differences in numbers captured in net traps (Table 5). Eighteen groups were more numerous in summer samples, and 17 taxa were taken predominantly in winter samples. Miscellaneous calanoid copepods were captured in statistically greater numbers in both seasons depending upon the substratum sampled. Pseudocyclops spp., Metis holothuriae, carideans, isopods, Spadella cephaloptera, and tunicate larvae were captured in statistically equal numbers from all substrata in both seasons.

Comparisons of emergence into net traps from different substrata within season and taxon indicated that 22 taxa emerged in significantly greater numbers from one of the 2 structurally complex substrata (coral or grass) either alone or in combination with one of the simple substrata (rubble or mud) (Table 6). A total of 10 taxa displayed significant differences in emergence but did not exhibit a clear pattern with regard to type of bottom. Copepodites of the calanoid Pseudodiaptomus mertoni, Pseudocyclops spp., copepod nauplii, carideans, caridean/anomuran larvae, miscellaneous nauplii, Spadella cephaloptera, chaetognaths in the genus Sagitta, gastropod veligers, and tunicate larvae emerged in statistically equal numbers from all 4 substrata. 
Table 3. Taxa displaying significant differences in numbers captured in re-entry traps among nights of exposure. Values are mean nos. $\mathrm{m}^{-2}(n=4)$; means are back-transformed from $\log (c+1)$; underlined means are significantly greater within taxon and season (ANOVA and S-N-K tests, $p<0.05$ )

\begin{tabular}{|c|c|c|c|}
\hline \multirow[t]{2}{*}{ Taxan/seasoh } & \multicolumn{3}{|c|}{ Night } \\
\hline & 1 & 2 & 3 \\
\hline \multicolumn{4}{|l|}{ Acartıö spp adults } \\
\hline & 0 & 124.0 & 62.1 \\
\hline Winter & 399.2 & 92.3 & $\overline{5.3}$ \\
\hline \multicolumn{4}{|l|}{ Acartia spp copepodites } \\
\hline \multirow{2}{*}{\multicolumn{4}{|c|}{ Miscellaneous calanoids }} \\
\hline & & & \\
\hline Winter & 56.0 & 0 & 3.5 \\
\hline \multicolumn{4}{|l|}{ Oithona spp. } \\
\hline Summer & 323.7 & 7004.2 & 5433.9 \\
\hline \multicolumn{4}{|l|}{ Miscellaneous cyclopoids } \\
\hline & 67.9 & 151.9 & 0 \\
\hline
\end{tabular}

\section{Pooled rotary emergence trap data}

ANOVA applied to RET data that had been summed across the 14 sampling intervals indicated a significant interaction effect due to season, lunar period, substratum, and taxon (Cochran's value unadjusted = $0.023, \mathrm{Df}=1,1032, p<0.05 ;$ Cochran's value adjusted $=0.0113, p>0.05$, loss of $12 \mathrm{Df}_{\mathrm{i}} F=2.32$, Df $=$ $252,1020, p<0.001)$. Of the 43 taxa included in the analysis, only Oithona spp., miscellaneous harpacticoids, copepod nauplii, and bivalve veligers were consistently taken in greater numbers as shown by S-N-K multiple comparisons ( $\mathrm{SE}=0.15$ ).

S-N-K tests among mean numbers of zooplankters emerging $\mathrm{m}^{-2}$ night $^{-1}$ revealed significant seasonal differences for all 43 taxa (Table 7), significant lunar differences for 40 groups (Table 8 ), and significant substratum-related differences for 42 taxa (Table 9). Summer increases in emergence were observed for 25 taxa, and winter increases in numbers were indicated for 7 taxa. Eleven groups did not display a consistent seasonal pattern. Fifteen taxa emerged in statistically greater numbers in either lunar quarter or the new moon, that is during lunar periods in which at least part of the night is moonless. Another set of 15 taxa displayed significantly greater emergence during full moons and no more than 2 of the other 3 lunar periods. Ten of the remaining 13 groups were captured in significantly greater numbers in each of the 4 lunar periods depending upon the season and substratum sampled. Oithona spp., miscellaneous harpacticoids, and gastropods emerged in statistically equal numbers during all lunar periods. Samples taken over coral and grass yielded greater numbers of 24 taxa, and a further 10 groups emerged in greater numbers from either complex substratum in combination with mud. Eight taxa did not display a consistent substratum-related
Table 4. Taxa displaying significant seasonal differences in numbers captured in re-entry traps. Values are mean nos. $\mathrm{m}^{-2}$ $(n=6)$; means are back-transformed from $\log (c+1)$; underlined means are significantly greater within taxon and night

(ANOVA and S-N-K tests, $p<0.05$ ). cop.: copepodites

\begin{tabular}{|c|c|c|}
\hline \multirow[t]{2}{*}{ Taxon/night } & \multicolumn{2}{|c|}{ Season } \\
\hline & Summer & Winter \\
\hline \multicolumn{3}{|c|}{ Summer $>$ Winter } \\
\hline \multicolumn{3}{|c|}{ Pseudodiaptomus coletaxi od } \\
\hline Night 2 & 352.4 & 27.8 \\
\hline Night 3 & 196.1 & 3.5 \\
\hline \multicolumn{3}{|c|}{ Pseudodiaptomus colefaxi ovigerous $P Q$} \\
\hline Night 2 & 278 & 0 \\
\hline \multicolumn{3}{|c|}{ Pseuciodraptomus colefaxi cop } \\
\hline Nighe 3 & 435.8 & 27.8 \\
\hline \multicolumn{3}{|c|}{ Miscellaneous cälanolds } \\
\hline Night 2 & 155.6 & 0 \\
\hline Night 3 & 58.8 & 3.5 \\
\hline \multicolumn{3}{|l|}{ Calànoid cop } \\
\hline Night 2 & 277.4 & 19.6 \\
\hline Night 3 & 87.7 & 5.3 \\
\hline \multicolumn{3}{|l|}{ Oithona spp. } \\
\hline Night 2 & 7004.2 & 391.0 \\
\hline Night 3 & 5433.9 & 131.6 \\
\hline \multicolumn{3}{|c|}{ Miscellaneous harpacticoids } \\
\hline Night 2 & 69168.7 & 1898.9 \\
\hline Night 3 & 85053.7 & 1358.5 \\
\hline \multicolumn{3}{|c|}{ Copepod nauplii } \\
\hline Night 2 & 3594.2 & 68.8 \\
\hline Night 3 & 3185.9 & 87.7 \\
\hline \multicolumn{3}{|l|}{ Ostracods } \\
\hline Night 3 & 21525 & 138.7 \\
\hline \multicolumn{3}{|c|}{ Cimpede larvae: } \\
\hline Night 1 & 166.4 & 5.3 \\
\hline Night 2 & 44.2 & 0 \\
\hline \multicolumn{3}{|l|}{ Polychaetes } \\
\hline Night 3 & 260.1 & 35 \\
\hline \multicolumn{3}{|c|}{ Polychaete larvae } \\
\hline Night 3 & 194.1 & 5.3 \\
\hline \multicolumn{3}{|l|}{ Sagitta spp. } \\
\hline Night 2 & 39.2 & 0 \\
\hline Night 3 & 19.6 & 0 \\
\hline \multicolumn{3}{|l|}{ Gastropods } \\
\hline Night 1 & 447.5 & 5.3 \\
\hline \multicolumn{3}{|c|}{ Gastropod veligers } \\
\hline Night 1 & 39.4 & 0 \\
\hline Night 2 & 348.0 & 3.5 \\
\hline Night 3 & 133.8 & 5.3 \\
\hline \multicolumn{3}{|c|}{ Winter > Summer } \\
\hline \multicolumn{3}{|c|}{ Acartia spp. adults } \\
\hline Night 1 & 0 & 399.2 \\
\hline Acartia spp. 0 & & \\
\hline Night 1 & 0 & 89.0 \\
\hline Parategastes & & \\
\hline Night 1 & 5.3 & 75.9 \\
\hline Night 2 & 0 & 136.0 \\
\hline Night 3 & 19.6 & 232.6 \\
\hline Summer $=W_{i}$ & & \\
\hline Miscellaneous & & \\
\hline Night 1 & 67.9 & 5.3 \\
\hline Night 3 & 0 & 48.0 \\
\hline
\end{tabular}

pattern in emergence, and copepod nauplii emerged in statistically equal numbers from all types of bottom.

\section{Unpooled rotary emergence trap data}

Forty-three separate ANOVAs, one per taxon, were used to analyse unpooled RET data. A total of 34 taxa 
Table 5. Taxa displaying significant seasonal differences in numbers captured in net emergence traps. Values are mean nos. $\mathrm{m}^{-2}$ night $^{-1}(n=12)$; means are back-transformed from $\log (c+1)$; underlined means are significantly greater within taxon and substratum (ANOVA and S-N-K tests, $p<0.05$ ). cop.; copepodites $;=$ : means are equal

\begin{tabular}{|c|c|c|c|c|c|c|c|c|}
\hline \multirow[t]{2}{*}{ Taxon } & \multicolumn{2}{|c|}{ Coral } & \multicolumn{2}{|c|}{ Rubble } & \multicolumn{2}{|c|}{ Grass } & \multicolumn{2}{|c|}{ Mud } \\
\hline & Summer & Winter & Summer & Winter & Surnmer & Winter & Summer & Winter \\
\hline \multicolumn{9}{|l|}{ Summer $>$ Winter } \\
\hline Pseudodiaptomus mertoni adults & & & & & 1.7 & 0 & & \\
\hline Pseudodiaptomus mertoni cop & & & & $=$ & 1.6 & 0.2 & & \\
\hline Ridgewayia spp & 9.2 & 0 & 4.6 & 0.1 & & $=$ & & \\
\hline Oithons spp. & & & 30.1 & 668 & 109.4 & 10.1 & 495. & 16.0 \\
\hline Miscellaneous cyclopoids & & & & & 9.6 & 0.9 & 10.2 & 1.5 \\
\hline Parategastes sp. & 11.7 & 5.6 & & & 537 & 125.4 & 20.4 & 6.7 \\
\hline Peltidium spp. & 2.3 & 0.3 & & $=$ & & $=$ & & \\
\hline Cumaceans & 2.1 & 0.4 & & & & $=$ & & \\
\hline Mysids & 2.6 & 0.6 & 2.7 & 0.7 & & $=$ & 1.5 & 4.7 \\
\hline Tanaids & & & & & 4.4 & 1.7 & 1.9 & 0.2 \\
\hline Miscellaneous naupiı & & & & & 2.6 & 0 & 1.6 & 0.1 \\
\hline Zoeae & 15.8 & 0 & 1.0 & 01 & & $=$ & 1.1 & 0 \\
\hline Megalopa & & & & & 4.1 & 0.6 & & \\
\hline Caridean/anomurañ làrvâe & 2.6 & 02 & & & $\overline{49}$ & 0.2 & 6.5 & 11 \\
\hline Sagutia spp. & 1.7 & 0.2 & & & 1.8 & 0 & 2.3 & 0.1 \\
\hline Polychaetes & & & & & 8.3 & 1.5 & 3.7 & 0.9 \\
\hline Gastropods & 136 & 2.0 & 77 & 32 & 93.8 & 12.7 & 15.7 & 16 \\
\hline Gastropod veligers & 61.9 & 284 & & & 62.2 & 32.7 & & \\
\hline \multicolumn{9}{|l|}{ Winter $>$ Summer } \\
\hline Pseudodiaptomus colefaxi d है & 2.3 & 31.9 & 22 & 46.6 & 523 & 9.7 & 12.7 & 26.6 \\
\hline Pseudodiaptomus colefaxi? & 1.3 & 7.0 & 0.4 & 7.4 & 0.2 & 0.5 & & \\
\hline Pseudodiaptomus colefaxj ovigerous $9 \mathrm{z}$ & 1.2 & 19.3 & 1.0 & 33.0 & 5.8 & 1.3 & 2.0 & 72 \\
\hline Pseudodiaptomus colefaxi cop. & 2.0 & 19.0 & 1.1 & 19.0 & \multicolumn{2}{|c|}{$=$} & & \\
\hline Stephos spp. & 10 & 12.6 & 03 & 6.5 & \multicolumn{2}{|c|}{$=$} & 1.2 & 3.4 \\
\hline Acartia spop. adults & 42 & $\frac{1.0}{47.5}$ & 5.2 & 48.3 & \multirow{2}{*}{\multicolumn{2}{|c|}{$\begin{array}{l}= \\
=\end{array}$}} & \multicolumn{2}{|c|}{$=$} \\
\hline Acartia spp. cop. & 74 & 105.7 & 9.6 & 122.0 & & & & \\
\hline Parvocalanus spp. & 39 & 17.6 & 53 & 21.9 & \multicolumn{2}{|c|}{$=$} & \multicolumn{2}{|c|}{$=$} \\
\hline Calanoid cop. & $9 \mathrm{l}$ & $\frac{1.1 .3}{41.3}$ & 7.8 & $\frac{2.0}{28.9}$ & \multicolumn{2}{|c|}{$=$} & 8.1 & $=$ \\
\hline Miscellaneous harpacticolds & 68.9 & 125.4 & 61.2 & 121.8 & \multicolumn{2}{|c|}{$=$} & 226.8 & 89.3 \\
\hline Copepod nauplii & 671 & 374.3 & 38.6 & 313.5 & \multicolumn{2}{|c|}{$=$} & 1031 & 2377 \\
\hline Amphipods & & & & & 104 & 54.6 & 3.0 & 15.4 \\
\hline Ostracods & 41 & 89 & 31 & 14.6 & \multicolumn{2}{|r|}{ 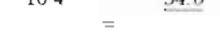 } & \multicolumn{2}{|c|}{$\cong$} \\
\hline Cinipede larvae & 13 & 39.6 & 1.1 & 147 & 7.1 & 0.9 & 1.2 & $\underline{36}$ \\
\hline $\begin{array}{l}\text { Larvaceans } \\
\text { a de }\end{array}$ & 29 & $\frac{290}{290}$ & 2.1 & 27.5 & 4.0 & 0.8 & 1.2 & $\underline{-1}$ \\
\hline Polychaete larvae & 6.2 & 16.7 & 2.6 & 35.4 & \multicolumn{2}{|c|}{$=$} & \multicolumn{2}{|c|}{$\begin{array}{l}= \\
=\end{array}$} \\
\hline Bivalve veligers & 873 & 1163.7 & 123.5 & 1272.1 & & $=$ & 1084 & 341.3 \\
\hline \multicolumn{9}{|l|}{ Summer $=$ Winter } \\
\hline Miscellaneous calanoids & 26 & 8.2 & 40 & 92 & 17.6 & 0.8 & 10.2 & 1.7 \\
\hline
\end{tabular}

exhibited significant effects. In 28 cases, a significant interaction was found among season, lunar period, substratum, and time of first emergence. Five groups had significant interaction effects at other levels, and a main effect due to time of first emergence was revealed for gastropod veligers. Of the 34 taxa with significant effects due to time of first emergence, 28 had peaks in emergence of greater than 10 zooplankters $\mathrm{m}^{-2}$ $90 \mathrm{~min}^{-1}$. Temporal patterns in emergence in these 28 taxa were assigned to 5 categories (Table 10, Fig. 1). Sunset emergence (Fig. 1a, b), a sharp peak in numbers captured near twilight, was the most common pattern with 56 occurrences in 14 taxa. Nocturnal migration (Fig. 1c) with maximum emergence covering a broad part of the night or dissociated from sunset and sunrise appeared 18 times in 12 taxa. A sharp peak in emergence near sunrise was observed once for polychaetes (Fig. 1d). A bimodal pattern (Fig. 1e, f), 2 peaks in emergence within 1 night, was recorded on 9 occasions for 5 different groups. Nine taxa displayed a diumal pattern (Fig. 1g, h), maximum emergence during the daytime, in 13 sets of samples. The 6 groups with peaks of emergence of less than 10 individuals $\mathrm{m}^{-2} 90 \mathrm{~min}^{-1}$ were characterized by diumal emergence in 3 cases Metis holothuriae, tanaids, and tunicate larvae - and nocturnal emergence for carideans, isopods, and Spadella cephaloptera.

Emergence patterns in 9 taxa with statistically equal numbers emerging $\mathrm{m}^{-2} 90 \mathrm{~min}^{-1}$ were classified by examination of the data. Harpacticoid copepods in the genus Peltidium exhibited sunset emergence (18:00 to $22: 30 \mathrm{~h}, 46 \%$ of the total number captured). Another harpacticoid, Parategastes sp., emerged mainly at night (18:00 to $01: 30 \mathrm{~h}, 68 \%$ of the total number), Diurnal emergence was exhibited by Acartia spp. copepodites, calanoids in the genus Parvocalanus, calanoid copepodites, miscellaneous cyclopoid copepods, cirripede larvae, miscellaneous nauplii, and gastropods $(15: 00$ to $18: 00 \mathrm{~h}$ and $04: 30$ to $12: 00 \mathrm{~h}$. between 31 and $71 \%$ of the total number).

The presence of interaction effects in most ANOVAs performed on unpooled RET data meant each taxon exhibited significant temporal patterns in emergence only during certain sampling periods. The distribution 
Table 6. Taxa displaying significant differences in numbers captured in net emergence traps among substrata. Values are mean nos. $\mathrm{m}^{-2}$ night ${ }^{-1}(n=12)$; means are back-transformed from $\log (c+1)$; underlined means are significantly greater within taxon and season (ANOVA and S-N-K tests, $p<0.05$ ). cop.: copepodites; $=$. means are equal

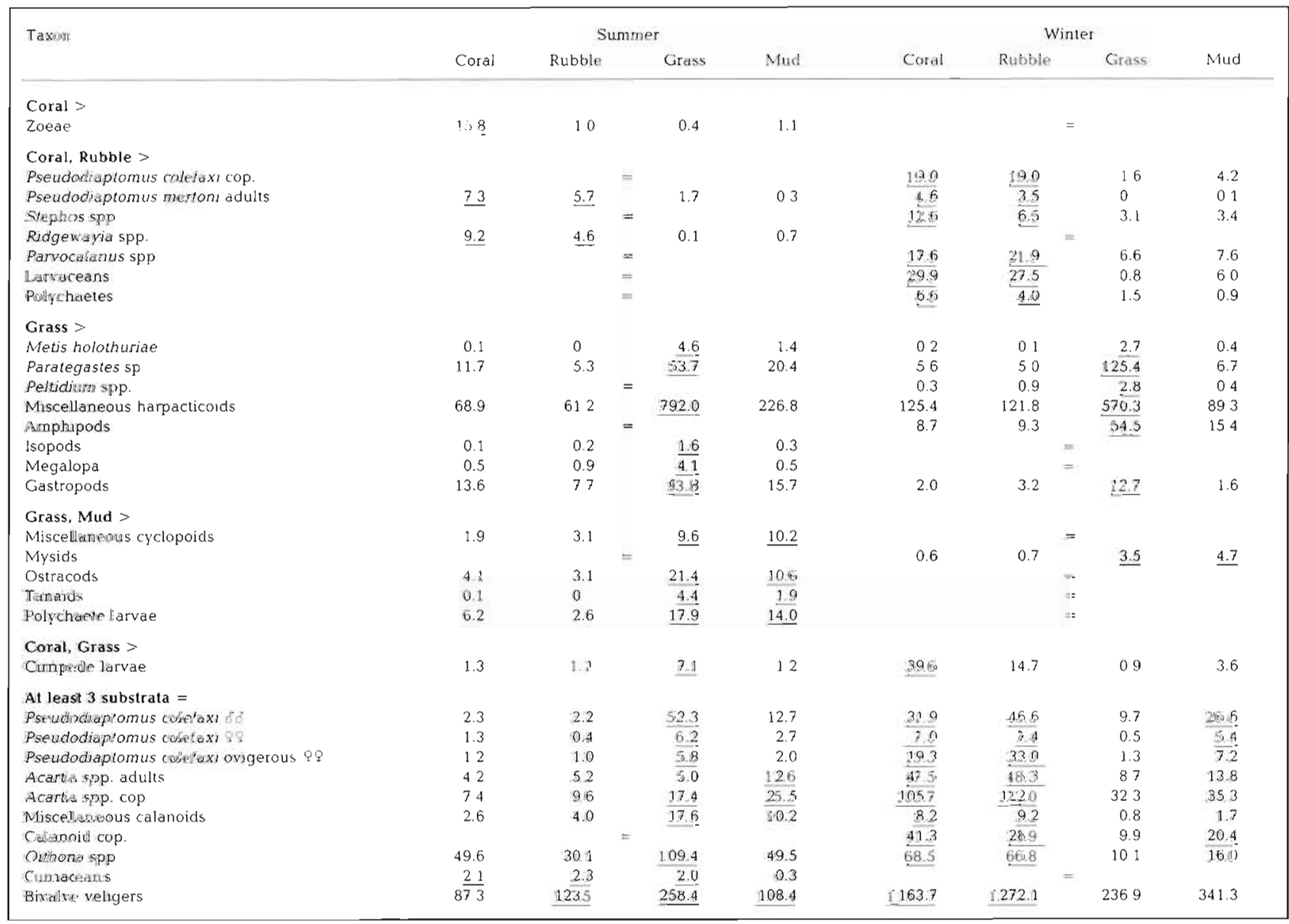

of significant temporal patterns with peaks in emergence of more than 10 individuals was relatively even between seasons, among lunar periods, and among substrata (Table 11). Zooplankters emerged in sufficient numbers to generate statistically significant temporal patterns during all sampling periods. In addition, emergence peaks were observed during all phases of the tide (Fig. 1).

Ten taxa displayed more than one pattern in emergence (Table 10, Fig. 1). Typically, the patterns exhibited were sunset emergence, nocturnal emergence, and bimodal emergence. Variations in temporal patterns tended to be consistent for age and sex classes within the 2 species of Pseudodiaptomus (Table 10, Fig. $1 \mathrm{a}, \mathrm{b}, \mathrm{e}, \mathrm{f})$.

Multiple comparisons applied to unpooled RET data established or confirmed seasonal differences in emergence for various taxa. Pseudocyclops spp., miscellaneous calanoid copepods, Metis holothuriae, miscellaneous harpacticoids, carideans, isopods, ostracods, tanaids, miscellaneous nauplii, Spadella cephaloptera, larvaceans, polychaetes, polychaete larvae, bivalve veligers, and tunicate larvae were shown to have emerged in greater numbers in the summer. Acartia spp. copepodites, calanoid copepodites, miscellaneous cyclopoid copepods, Parategastes sp., and copepod nauplii were more numerous in winter samples. All 4 groups of Pseudodiaptomus colefaxi, as well as cirripede larvae, emerged in nearly equal numbers during both seasons.

Variations in emergence among lunar periods were indicated by some ANOVAs. Pseudodiaptomus colefaxi females and copepodites, Acartia spp. adults, Peltidium spp., miscellaneous harpacticoids, and mysids emerged in greater numbers during either lunar quarter or the new moon. Statistically significant emergence during the full moon was revealed for $P$. colefaxi males and ovigerous females, Oithona spp., and chaetognaths in the genus Sagitta. Equal emergence in all 4 lunar periods was indicated for Parvocalanus sp., calanoid copepodites, amphipods, cumaceans, and gastropods.

Analysis of unpooled RET data clarified substratum- 
Table 7. Taxa displaying significant seasonal differences in numbers captured in RETs. Values are mean nos. $\mathrm{m}^{-\cdots}$ night ${ }^{-1}(n=2)$; means are back-transformed from $\log (c+1)$; underlined means are significantly greater within taxon, lunar period, and substratum (ANOVA and S-N-K tests, $p<0.05$ ). FQ: first quarter; FM: full moon; LQ: last quarter; NN: new moon; cop.: copepodites; $=$ : means are equal

\begin{tabular}{|c|c|c|c|c|c|c|c|c|}
\hline \multirow[t]{2}{*}{ Taxon/substratum } & \multicolumn{2}{|c|}{$F Q$} & \multicolumn{2}{|c|}{$F M$} & \multicolumn{2}{|c|}{ LQ } & \multicolumn{2}{|c|}{ NM } \\
\hline & Summer & Winter & Surnmer & Winter & Summer & Winter & Summer & Winter \\
\hline \multicolumn{9}{|c|}{ Summer $>$ Winter } \\
\hline \multicolumn{9}{|c|}{ Pseudadiaptomus colefaxi of } \\
\hline Coral & \multicolumn{2}{|c|}{$=$} & & & 250 & 78 & & \\
\hline Grass & 55.4 & 289.1 & 278.2 & 38.0 & 311.1 & 55.9 & 175.4 & 53.5 \\
\hline Mud & 11.7 & $\overline{151.4}$ & & & 304.2 & 31.8 & & \\
\hline Pseudodiaptomus me & & & & & & & & \\
\hline Coral & $\underline{238.9}$ & 1.8 & 73.1 & 2.5 & 101.3 & 1.4 & & \\
\hline Grass & 99.0 & 0.4 & 29.2 & 0.7 & 34.5 & 0 & 13.8 & 1.0 \\
\hline Mud & 11.9 & 0 & 7.3 & 0.4 & 11.6 & 0.7 & 4.2 & 1.0 \\
\hline Pseudodiaptomus me & & & & & & & & \\
\hline Coral & 140.2 & 1.0 & 48.0 & 2.9 & $\underline{57.9}$ & 1.0 & 40.7 & 64 \\
\hline Grass & 96.7 & 1.4 & 39.7 & 0 & 32.1 & 0 & 30.6 & 0 \\
\hline Mud & $\overline{15.8}$ & 0.4 & 5.3 & 0.4 & 9.5 & 0 & & \\
\hline Pseudodiaptomus me & & & & & & & & \\
\hline Coral & 554.0 & 0.4 & 197.1 & 1.0 & 253.3 & 0.4 & 147.1 & 10.6 \\
\hline Grass & 84.1 & 1.4 & 123.7 & 0.4 & 196.1 & 0 & 1062 & 1.0 \\
\hline Mud & $\overline{51.7}$ & 16 & 47.0 & 0.4 & 1024 & 0 & 378 & 0 \\
\hline Ridgewayid spp. & & & & & & & & \\
\hline Coral & 14.6 & 2.2 & 53.7 & 04 & 40.6 & 0.4 & $\underline{18,0}$ & 0.4 \\
\hline Grass & & & 95 & 04 & 12.3 & 1.4 & $\overline{104}$ & 0.7 \\
\hline Mud & & & 4.7 & 0 & 9.2 & 0 & 1.8 & 0 \\
\hline Miscellaneous calano & & & & & & & & \\
\hline Coral & 428.4 & 86.0 & 99.4 & 11.0 & 6293 & 5.7 & & \\
\hline Grass & 154.9 & 449 & 168.2 & 4.9 & 504.0 & 74 & 72.5 & 6.4 \\
\hline Mud & & & 104.4 & 8.9 & 282.0 & 4.9 & 36.8 & 5.7 \\
\hline Oithona spp. & & & & & & & & \\
\hline Coral & 3240.4 & 830.5 & 2830.4 & 667.5 & 2476.0 & 491.3 & & \\
\hline Grass & $3640 . \overline{7}$ & 884.9 & $\overline{3288.1}$ & 446.6 & 3058.3 & 552.3 & 1839.0 & 392.9 \\
\hline Mud & 3216.8 & 843.1 & $3 \overline{49.3}$ & 591.2 & 2315.7 & 359.9 & 1676.2 & 441.7 \\
\hline Metis holothuriae & & & & & & & & \\
\hline Coral & & & & & & & 6.3 & 0 \\
\hline Grass & 24.1 & 92.2 & 30.7 & 8.9 & & & 99.1 & 10.7 \\
\hline Mud & 2.7 & 11.5 & 35.9 & 89 & & & 44.7 & 3.2 \\
\hline Peltidium spp. & & & & & & & & \\
\hline Coral & 160 & 2.9 & 7.5 & 07 & & & 36.0 & 20 \\
\hline Grass & 10.0 & 47.4 & $1 \overline{5.6}$ & 4.9 & & & 91.0 & 30 \\
\hline Mud & 2.2 & 7.8 & 105 & 0.7 & & & 92 & 2.2 \\
\hline Miscellaneous harpad & & & & & & & & \\
\hline Coral & 3177.8 & 744.8 & 27889 & 897.2 & 3670.0 & 785.1 & & \\
\hline Mud & & & & & 2655.8 & 395.3 & & \\
\hline Carideans & & & & & & & & \\
\hline Coral & 8.8 & 0.4 & & & 5.0 & $a$ & & \\
\hline Grass & 29.6 & 1.0 & 8.8 & 0 & 8.7 & 1.2 & 3.0 & 0 \\
\hline Mud & & & 2.5 & 0 & 121 & 2.9 & & \\
\hline Cumaceans & & & & & & & & \\
\hline Coral & & & 46.7 & 74 & 43.5 & 74 & 239 & 4.3 \\
\hline Grass & & & 89.5 & 63 & & & & \\
\hline Mud & 0 & 13.1 & & & & & & \\
\hline Isopods & & & & & & & & \\
\hline Coral & & & & & & & $\underline{4.8}$ & 0 \\
\hline Grass & 27 & 0.4 & $\underline{5.3}$ & 0.4 & & & & \\
\hline Mud & & & & & & & 7.4 & 0 \\
\hline Mysids & & & & & & & & \\
\hline Coral & 145 & 3.2 & 62.1 & 7.5 & 65.1 & 65 & 82.9 & 5.0 \\
\hline Grass & 42.9 & 28.8 & 56.2 & 35 & & & & \\
\hline Ostracods. & & & & & & & & \\
\hline Coral. & & & 33.5 & 100 & 87.3 & 15.5 & 1189 & 1.8 \\
\hline Grass & 446.9 & 14.5 & 92.4 & 39 & 185.3 & 79 & 370.1 & 3.6 \\
\hline Mud & 1167 & 10.5 & 69.0 & 45 & 291.4 & 20 & 279.1 & 45 \\
\hline Zoeae & & & & & & & & \\
\hline Coral & 1372 & 6.5 & 533 & 3.9 & 22.7 & 5.0 & 77.9 & 5.3 \\
\hline Grass & 19.0 & 2.5 & 11.5 & 392 & 494 & 15.2 & 59.5 & 14.2 \\
\hline Mud & 147 & 3.0 & & & 26.0 & 4.0 & & \\
\hline Megalopa & & & & & & & & \\
\hline Coral & & & & & & & 2.0 & 0 \\
\hline Grass & & & 200.5 & 2.0 & 5.9 & 1.0 & 7.8 & 1.4 \\
\hline Mữ & 0.4 & 7.8 & & & & & 4.5 & 15.7 \\
\hline Caridean/anomuran I & & & & & & & & \\
\hline Coral & 19.5 & 0 & 27.6 & 0 & 1327 & 0 & 43.1 & 0 \\
\hline Grass & 24.1 & 0.7 & 80.6 & 8.8 & $93 i$ & 0 & 80.0 & 1.4 \\
\hline Mud & 9.2 & 1.8 & 92.7 & 13.9 & 51.3 & 10 & 50.6 & 1.0 \\
\hline
\end{tabular}


Table 7 (continued)

\begin{tabular}{|c|c|c|c|c|c|c|c|c|}
\hline \multirow[t]{2}{*}{ Taxon/substratum } & \multicolumn{2}{|c|}{$\mathrm{FQ}$} & \multicolumn{2}{|c|}{$F M$} & \multicolumn{2}{|c|}{ LQ } & \multicolumn{2}{|c|}{$\mathrm{NM}$} \\
\hline & Summer & Winter & Summer & Winter & Summer & Winter & Summer & Winte: \\
\hline \multicolumn{9}{|l|}{ Summer $>$ Winter } \\
\hline \multicolumn{9}{|l|}{ Spadella cephaloptera } \\
\hline Coral & 14.6 & 0.4 & & $=$ & & & $\frac{17.4}{26 .}$ & 1.0 \\
\hline Grass & 4.5 & 0.4 & 14.3 & 0.4 & & & 25.5 & 0 \\
\hline Mud & 4.7 & 0 & & $=$ & & & 30.5 & 0 \\
\hline \multicolumn{9}{|l|}{ Sagitta spp. } \\
\hline Coral & 184.4 & 1.8 & 70.2 & 1.8 & 397.7. & 1.8 & & \\
\hline Grass & 212.5 & 6.0 & 399.7 & 04 & 235.5 & 0.4 & 510 & 1.4 \\
\hline Mud & $\underline{309.3}$ & 2.5 & 255.8 & 14 & 267.1 & 0.7 & 29.5 & 0.4 \\
\hline \multicolumn{9}{|l|}{ Larvaceans } \\
\hline Coral & & & 761.0 & 435 & 1126.1 & 23.7 & 181.3 & 10350.4 \\
\hline Grass & 46.2 & 11.7 & 326.9 & 724 & 10947 & 3.6 & 187.5 & 2166.5 \\
\hline Mud & 88.5 & 25.6 & 364.9 & 77.9 & 871.1 & 5.6 & 34.0 & .1448 .6 \\
\hline \multicolumn{9}{|l|}{ Polychaete larvae } \\
\hline Coral & 287.0 & 85.5 & & $=$ & & & & \\
\hline Grass & & & & $=$ & 328.9 & 85.3 & & \\
\hline Mud & & & & $=$ & 377.8 & 101.3 & & \\
\hline \multicolumn{9}{|l|}{ Gastropods } \\
\hline Coral & 487.9 & 0.7 & 349.2 & 0 & $\underline{123.5}$ & 0.4 & $\underline{225.1}$ & 0.4 \\
\hline Grass & 741.8 & 8.4 & 740.9 & 17.4 & 385.5 & 5.3 & 571.7 & 12.4 \\
\hline Mud & 207.4 & 2.9 & 199.0 & 14.3 & $\underline{272.9}$ & 0 & 86.4 & 6.5 \\
\hline \multicolumn{9}{|l|}{ Bivalve veligers } \\
\hline Corâl & 3833.3 & 7.0 & 5291.4 & 137.4 & 1550.4 & 217.9 & 1086.6 & 4752.9 \\
\hline Grass & 1210.3 & 16.9 & 1609.1 & 133.9 & 4349.4 & 128.4 & & \\
\hline Mud & 377.4 & 04 & 772.1 & 1281 & 1293.1 & 64.5 & 435.8 & 1247.2 \\
\hline Tunicate larvae & & & & & & & & \\
\hline Coral & & & & $=$ & & & $\underline{3.0}$ & 0.4 \\
\hline Grass & & & & $=$ & & & 3.6 & $a$ \\
\hline Mud & & & $\underline{7.4}$ & 0.4 & $\underline{23.5}$ & 3.2 & 3.9 & 0.4 \\
\hline Winter $>$ Summer & & & & & & & & \\
\hline Acartia spp. adults & & & & & & & & \\
\hline Coral & & & 14.9 & 244.4 & 1378.7 & 106.2 & 40.8 & 17040.9 \\
\hline Grass & 348.9 & 65.4 & & $=$ & & & 874 & 407.7 \\
\hline Mud & 112.9 & 363.3 & & $=$ & & & 32.2 & 532.0 \\
\hline Stephos spp. & & & & & & & & \\
\hline Coral & 1.8 & 39.7 & 3.0 & $\underline{39.4}$ & 2.2 & 44.5 & 3.5 & 15.5 \\
\hline Grass & 11.8 & $\underline{36.1}$ & & $=$ & & & & \\
\hline Mud & 2.2 & 7.5 & & $=$ & & & & \\
\hline Parvocalanus sp. & & & & & & & & \\
\hline Coral & 341.5 & 1053.4 & 199.2 & $\underline{525.7}$ & & & 182.2 & 1031.7 \\
\hline Mud & & & & & 547.8 & 86.7 & 120.6 & 499.8 \\
\hline Miscellaneous cyclopo & & & & & & & & \\
\hline Coral & & & 63.7 & 318.5 & & & 98.5 & 525.1 \\
\hline Grass & & & 52.7 & 248.0 & & & & \\
\hline Mud & & & 35.1 & 235.5 & & & 49.6 & 332.6 \\
\hline Parategastes sp. & & & & & & & & \\
\hline Coral & & & & & 85.8 & 320.1 & & \\
\hline Grass & 657.6 & 10207.2 & 651.9 & 1724.9 & 412.4 & 2152.7 & & \\
\hline Mud & 56.3 & $\underline{243.5}$ & & $=$ & 32.0 & 103.6 & & \\
\hline Summer $=$ Winter & & & & & & & & \\
\hline Amphipods & & & & & & & & \\
\hline Coral & 27.6 & $\underline{87.4}$ & & $=$ & & & & \\
\hline Grass & 70.4 & 379.9 & & $=$ & & & & \\
\hline Mud & 4.9 & $\underline{104.5}$ & & $=$ & & & 28.2 & 80.7 \\
\hline Gastropod veligers & & & & & & & & \\
\hline Coral & & & 87.5 & $\underline{550.1}$ & & & 299.9 & $\underline{1485.9}$ \\
\hline Grass & & & 87.3 & 690.5 & 806.0 & 208.7 & & \\
\hline Mud & & & 106.3 & 375.9 & & & & \\
\hline Pseudodiaptomus cole & & & & & & & & \\
\hline Grass & 14.5 & $\underline{60.9}$ & $\underline{82.2}$ & 7.1 & $\underline{84.0}$ & 20.3 & & \\
\hline Mud & 2.9 & $\underline{36.8}$ & & & $\underline{84.0}$ & 20.3 & 24.4 & $\underline{67.5}$ \\
\hline Pseudodiaptomus cole & & & & & & & & \\
\hline Grass & 16.3 & 103.1 & 110.6 & 5.5 & 127.7 & 24.1 & $\underline{59.5}$ & 16.0 \\
\hline Mud & 6.7 & 40.8 & & & $\underline{125.4}$ & 9.1 & 1.8 & $\underline{13.5}$ \\
\hline
\end{tabular}


Table 7 (continued)

\begin{tabular}{|c|c|c|c|c|c|c|c|c|}
\hline \multirow[t]{2}{*}{ Taxon/substratum } & \multicolumn{2}{|c|}{$\mathrm{FQ}$} & \multicolumn{2}{|c|}{$F M$} & \multicolumn{2}{|c|}{$\mathrm{LQ}$} & \multicolumn{2}{|c|}{ NM } \\
\hline & Summer & Winter & Summer & Winter & Summer & Winter & Summer & Winter \\
\hline \multicolumn{9}{|c|}{ Summer $=$ Winter } \\
\hline \multicolumn{9}{|c|}{ Pseudodiaptomus rolefaxi cop } \\
\hline Coral. & \multicolumn{2}{|c|}{$=$} & \multicolumn{2}{|c|}{$=$} & 93.8 & 78 & \multirow{2}{*}{\multicolumn{2}{|c|}{$\begin{array}{l}= \\
=\end{array}$}} \\
\hline Grass & 142 & 129.2 & \multirow{2}{*}{\multicolumn{2}{|c|}{0.9}} & \multicolumn{2}{|l|}{93.8} & & \\
\hline Mud & 5.9 & 125.4 & & & $157: 4$ & 208 & \multicolumn{2}{|c|}{$=$} \\
\hline \multicolumn{9}{|l|}{ Acartio spp. cop. } \\
\hline Coral & & & & $=$ & 2081.3 & 408.1 & \multirow{2}{*}{\multicolumn{2}{|c|}{104489}} \\
\hline Grass & 646.1 & 282.5 & & $=$ & \multicolumn{2}{|c|}{$=$} & & \\
\hline Mud & 453.4 & 758.4 & & $=$ & 851.4 & 306.9 & 240.0 & 1541.2 \\
\hline \multicolumn{9}{|l|}{ Pseudocyclops spp. } \\
\hline Coral & \multicolumn{2}{|c|}{$=$} & \multirow[t]{3}{*}{320} & 5.9 & \multicolumn{2}{|c|}{$=$} & \multicolumn{2}{|c|}{$=$} \\
\hline Grass & 3.9 & 19.5 & & $=$ & 3.0 & $\underline{10.5}$ & & \\
\hline \multicolumn{8}{|l|}{ Calanoid cop } & \\
\hline Coral & \multirow{2}{*}{\multicolumn{2}{|c|}{$\begin{array}{l}= \\
=\end{array}$}} & & $=$ & \multicolumn{2}{|c|}{$=$} & 502.3 & 4190.1 \\
\hline Mud & & & & $=$ & 1178.6 & 289.9 & 2429 & 1279.4 \\
\hline \multicolumn{9}{|l|}{ Copepod nauplii } \\
\hline Coral & \multirow{2}{*}{\multicolumn{2}{|c|}{$=$}} & & $=$ & \multicolumn{2}{|r|}{$=$} & 17792 & 6286.2 \\
\hline Mud & & & & $=$ & 10240.3 & 10129 & & \\
\hline Tanaids & & & & & & & & \\
\hline Coral & & & 3.0 & 0.4 & & $=$ & & \\
\hline Grass & & & 35.7 & 9.5 & & $=$ & & \\
\hline Mud & 14 & 5.9 & & $=$ & & $=$ & & \\
\hline Miscellaneous nauplu & & & & & & & & \\
\hline Coral & 27.0 & 350.8 & & $=$ & & 8.9 & & 59 \\
\hline Grass & 20.0 & 157.8 & 73.9 & 24.5 & $\underline{92.3}$ & 12.0 & 26.5 & \\
\hline Mud & 5.0 & 203.1 & & 2as. & $\frac{2.6}{84.6}$ & & & \\
\hline Cimpede larvae & & & & & & & & \\
\hline Coral & & & & $=$ & 043.8 & 110.5 & 107.5 & 329.3 \\
\hline Grass & & & & $=$ & $589 \frac{2}{2}$ & 79.0 & & \\
\hline Mud & 6.0 & 35.9 & & $=$ & 4388 & 697 & & \\
\hline Polychaetes & & & & & & & & \\
\hline Coral & 22.4 & 2770 & 239 & 50 & & $=$ & & \\
\hline Mud & 8.9 & 292 & & $=$ & & $=$ & & \\
\hline
\end{tabular}

related emergence patterns for 34 taxa. All 3 groups of Pseudodiaptomus colefaxi adults, Stephos spp., carideans, mysids, ostracods, tanaids, caridean/anomuran larvae, zoeae, and Sagitta spp. were shown to have emerged in greater numbers from grass. Pseudodiaptomus mertoni males, females, and copepodites, Acartia spp. adults and copepodites, Pseudocyclops spp., Ridgewayia sp., Parvocalanus sp., miscellaneous calanoid copepods, calanoid copepodites, cirripede larvae, miscellaneous nauplii, larvaceans, polychaetes, polychaete larvae, gastropod veligers, and bivalve veligers were more numerous in samples taken above coral patches. Oithona spp., miscellaneous cyclopoid copepods, cumaceans, and Spadella cephaloptera were equally common in samples from coral and grass. Copepod nauplii and tunicate larvae were taken in equal numbers from all substrata.

\section{DISCUSSION}

Differences in sampling methodology and taxonomic classification have led to dispute over the importance of demersal zooplankton in various ecosystems (Alldredge \& King 1977, 1985, Porter et al. 1977, Hobson \& Chess 1979, Robichaux et al. 1981, Youngbluth 1982a). A definition of demersal zooplankton relies on an understanding of the animals' behavior, particularly in relation to the substratum. Emergence traps and reentry traps have been the primary tools used to sample demersal zooplankton. Both methods have biases including incidental trapping of non-demersal forms during deployment or recovery, capture of animals that crawl into traps, and contamination of emergence samples by zooplankters forced into traps through gaps between the bases and the bottom. In this study and a previous one (Jacoby \& Greenwood 1988), samples from net tows, re-entry traps, and emergence traps were compared in order to obtain a better idea of the distribution and behavior of various zooplankters.

Sixteen of the 43 common taxa in this study consisted of adults which were captured predominantly in reentry or emergence traps. These taxa displayed nighttime emergence and were rare in net tows or were clearly more common in night-time net tows. These groups (i.e. Pseudodiaptomus colefaxi males, females, and ovigerous females, Stephos spp., Pseudocyclops spp., Ridgewayia sp., Parategastes sp., Peltidium spp., amphipods, carideans, cumaceans, isopods, mysids, ostracods, Spadella cephaloptera, and polychaetes) were classified as demersal zooplankters. They were on or near the bottom during the day and emerged at night, although they may not have reached the surface of the water column in large numbers. This assemblage includes the largest zooplankters commonly captured.

Two other taxa, Pseudodiaptomus mertoni males and 
Table 8. Taxa displaying significant differences in numbers captured in RETs among lunar periods. Values are mean nos. $\mathrm{m}^{-2}$ night ${ }^{-1}(n=2)$; means are back-transformed from $\log (c+1)$; underlined means are significantly greater within taxon, season, and substratum (ANOVA and S-N-K tests, $p<0.05$ ). FQ: first quarter; FM: full moon; LQ: last quarter; NM: new moon; cop.: copepodites; $=\cdot$ means are equal

\begin{tabular}{|c|c|c|c|c|c|c|c|c|}
\hline \multirow[t]{2}{*}{ Taxon/substratum } & \multicolumn{4}{|c|}{ Summer } & \multicolumn{4}{|c|}{ Winter } \\
\hline & $F Q$ & $F M$ & LQ & NM & $\mathrm{FQ}$ & $\mathrm{FM}$ & $L Q$ & NM \\
\hline \multicolumn{9}{|c|}{ LQ. NM > } \\
\hline Coral & & & $=$ & & 0.4 & 1.0 & 04 & 106 \\
\hline Stephos spp. & & & & & & & & \\
\hline Mud & 2.2 & 3.5 & 7.5 & 26.5 & & & & \\
\hline \multicolumn{9}{|l|}{ Acartia spp. cop } \\
\hline \multicolumn{6}{|l|}{ Copepod nauplii } & 3712 & 408.1 & 16448.9 \\
\hline Mud & 1373.9 & 3117.6 & 10240.3 & 2055.0 & & & & \\
\hline \multicolumn{9}{|l|}{ Isopods } \\
\hline Coral & 0.7 & 04 & 0 & 4.8 & & & & \\
\hline Mud & 0.4 & 1.2 & 1.0 & $\overline{7.4}$ & & & & \\
\hline \multicolumn{9}{|l|}{ Bivalve veligers } \\
\hline Coral & & & $=$ & & 7.0 & 137.4 & 217.9 & 4752.9 \\
\hline Graas & 1210.3 & 16091 & 4349.4 & 863.7 & 16.9 & 133.9 & 128.4 & 935.6 \\
\hline Mud & & & 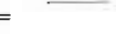 & & 0.4 & 128.1 & 64.5 & 1247.2 \\
\hline FQ, LQ, NM > & & & & & & & & \\
\hline Pseudodiaptomus merton & & & & & & & & \\
\hline Coral & & & $=$ & & 1.8 & 2.5 & 14 & 19.9 \\
\hline Grasss & 99.0 & 29.2 & 34.5 & 138 & & & & \\
\hline Mud & & & $=$ & & & & & \\
\hline Pseudodiaptomus mertoni & & & & & & & & \\
\hline Coral & & & $=$ & & & & & \\
\hline Grass & & & $=$ & & & & & \\
\hline Mud & & & $=$ & & 0.4 & 0.4 & 0 & 3.5 \\
\hline Miscellaneous calanodds & & & & & & & & \\
\hline Coral & 428.4 & 994 & 629.3 & 81.0 & 86.0 & 11.0 & 57 & 97.6 \\
\hline Grass & $\overline{154.9}$ & 1682 & 504.0 & 72.5 & $\overline{44.9}$ & 4.9 & 74 & 6.4 \\
\hline Mud & 174.2 & 104.4 & 282.0 & 36.8 & 154.8 & 8.9 & 49 & 5.7 \\
\hline Parategastes sp. & & & & & & & & \\
\hline Grass & & & $=$ & & 10207.2 & 17249 & 21527 & 960.2 \\
\hline Mud & 56.3 & 1317 & 32.0 & 351.5 & & & & \\
\hline Carideans & & & & & & & & \\
\hline Grass & 29.6 & 8.8 & 8.7 & 30 & & & & \\
\hline Mud & & & $=$ & & 3.9 & 0 & $\underline{2.9}$ & $\underline{3.5}$ \\
\hline Ostracods & & & & & & & & \\
\hline Coral & & & $=$ & & 46.4 & 106 & 15.5 & 1.8 \\
\hline Miscellaneous nauplii & & & & & & & & \\
\hline Coral & & & $=$ & & 350.8 & 14.2 & 24.1 & 54.0 \\
\hline Grass & 20.0 & 73.9 & 92.3 & 26.5 & 157.8 & 24.5 & 8.9 & 59 \\
\hline Mud & 5.0 & 25.5 & 84.6 & 25.1 & 203.1 & 26.3 & 12.0 & 387 \\
\hline Spàdella cephaloptera & & & & & & & & \\
\hline Coral & 14.6 & 1.0 & 2.2 & 17.4 & & & & \\
\hline Grass & $\overline{4.5}$ & 143 & 0 & 25.5 & & & & \\
\hline Mud & 4.7 & 1.4 & 0.4 & $\overline{30.5}$ & & & & \\
\hline Polychaetes & & & & & & & & \\
\hline Coral & 22.4 & 23.9 & 1722 & 35.0 & 277.0 & 5.0 & 1892 & 16.0 \\
\hline Grass & & & $=$ & & $90.0^{\circ}$ & 23.5 & 92.2 & 14.8 \\
\hline FM + Others > & & & & & & & & \\
\hline Pseudocyclops spp. & & & & & & & & \\
\hline Grass & 3.9 & 154 & 3.0 & 29 & & & & \\
\hline Ridgewayna spp & & & & & & & & \\
\hline Grass & 2.5 & 9.5 & 12.3 & 10.4 & & & & \\
\hline Mud & 0 & $\overline{4.7}$ & $\underline{9.2}$ & 1.8 & & & & \\
\hline Parvocalanus sp. & & & & & & & & \\
\hline Mud & & & $=$ & & 351.5 & 647.1 & 86.7 & 499.8 \\
\hline Calanoid cop. & & & & & & & & \\
\hline Coràl & & & $=$ & & 1496.9 & 795.3 & 1010.2 & $\mathrm{~A} 190.1$ \\
\hline Mud & & & $=$ & & 1121.1 & 817.5 & 289.9 & 1279.4 \\
\hline Metis holothuriae & & & & & & & & \\
\hline Coral & 5.0 & 0.4 & 0.7 & 6.3 & & & & \\
\hline Grass & & & $=$ & & $\underline{92.2}$ & 8.9 & 22.0 & 10.7 \\
\hline Mud & 2.7 & $\underline{35.9}$ & 79 & 44.7 & & & & \\
\hline Miscellaneous cyclopoids & & & & & & & & \\
\hline Mud & & & $=$ & & 22.8 & $\underline{235.5}$ & 72.9 & $32 \mathrm{z}$ \\
\hline Tanaids & & & & & & & & \\
\hline Mud & 1.4 & 16.3 & 2.5 & $\underline{11.8}$ & & & & \\
\hline Zoeae & & & & & & & & \\
\hline Grass & & & $=$ & & 2.5 & $\underline{39.2}$ & $\underline{152}$ & 14.2 \\
\hline Mud & & & $=$ & & 3.0 & $\underline{18.2}$ & $\overline{4.0}$ & $\overline{46.3}$ \\
\hline Megalopa & & & & & & & & \\
\hline Grass & 7.4 & 200.5 & 5.9 & 7.8 & 9.2 & 2.0 & 1.0 & 1.4 \\
\hline
\end{tabular}


Table 8 (continued)

\begin{tabular}{|c|c|c|c|c|c|c|c|c|}
\hline \multirow[t]{2}{*}{ Taxon/substratum. } & \multicolumn{4}{|c|}{ Summer } & \multicolumn{4}{|c|}{ Winter } \\
\hline & $\mathrm{FQ}$ & FM & LQ & NM & $F Q$ & FM & LQ & NM \\
\hline \multicolumn{9}{|c|}{ FM + Others > } \\
\hline \multicolumn{9}{|c|}{ Caridean/a nomuran larvae } \\
\hline $\begin{array}{l}\text { Coral } \\
\text { Grass }\end{array}$ & $\begin{array}{l}195 \\
241\end{array}$ & $\begin{array}{l}27.6 \\
80.6\end{array}$ & $\frac{132.7}{93.1}$ & $\begin{array}{l}43.1 \\
80.0\end{array}$ & 0.7 & 88 & 0 & 1.4 \\
\hline Mud & 9.2 & 92.7 & 51.3 & 50.6 & 18 & 139 & 1.0 & 1.0 \\
\hline \multicolumn{9}{|l|}{ Cimpede larvae } \\
\hline Coral & 27.9 & 62.9 & 943.8 & 107.5 & & & $=$ & \\
\hline Grass & 212 & 156.0 & 589.2 & 59.8 & 37.5 & 246.5 & 79.0 & 72.8 \\
\hline Mud & 60 & 83.3 & 438.8 & 27.7 & 35.9 & 212.7 & 697 & 52.9 \\
\hline \multicolumn{9}{|l|}{ Larvaceans } \\
\hline Coral & 34.3 & 761.0 & 1. 1.26 .1 & 1813 & 70.3 & 43.5 & 23.7 & 10350.4 \\
\hline Grass & 46.2 & $\overline{316.9}$ & 1094.7 & 1875 & 11.7 & 72.4 & 3.6 & 2166.5 \\
\hline Mud & 88.5 & 364.0 & 871.1 & 34.0 & 15.6 & 77.9 & 5.6 & 1.4480 \\
\hline \multicolumn{9}{|l|}{ Polychaete larvae } \\
\hline Grass & & & & & 324 & 318.2 & 85.3 & 205.8 \\
\hline \multicolumn{9}{|l|}{ Gastropod veligers } \\
\hline Coral & & & & & 81.0 & 550.1 & 424.3 & 14859 \\
\hline Grass & 92.4 & 87.3 & 8060 & 169.2 & 385 & 690.5 & 208.7 & 214.1 \\
\hline Mud & 34.0 & 106.3 & 2724 & 147.8 & 51.5 & 3759 & 106.9 & 3716 \\
\hline \multicolumn{9}{|l|}{ Tunicate larvae } \\
\hline Coral & 1.4 & 17.4 & 5.9 & 3.0 & 1.6 & 70 & 8.8 & 0.4 \\
\hline Grass & 1.8 & $\overline{2.0}$ & 13.1 & 3.6 & 20 & 4.5 & 10.5 & 0 \\
\hline Mud & 1.2 & 74 & 235 & 39 & 47 & 0.4 & 3.2 & 0.4 \\
\hline \multicolumn{9}{|c|}{ Lunar periods $=$} \\
\hline \multicolumn{9}{|c|}{ PSeudodiaptomus colefaxi } \\
\hline Coral & & & & & 36.8 & 80.1 & 7.8 & 545 \\
\hline Grass & 554 & 278.2 & 311.1 & 175.4 & 289.1 & 30.8 & 55.9 & 53.5 \\
\hline Mud & 11.7 & 148.7 & 304.2 & 21.0 & & & $=$ & \\
\hline \multicolumn{9}{|c|}{ Pseudodiaptomus coldaxi } \\
\hline Grass & 14.5 & 82.2 & 84.0 & 100.0 & 60.9 & 71 & 20.3 & 41.8 \\
\hline Mud & 29 & 46.4 & 84.0 & 24.4 & & & $=$ & \\
\hline Pseudodiaptomus cole & & & & & & & & \\
\hline Coral & & & & & 7.9 & $\underline{332}$ & 6.5 & 25.6 \\
\hline Grass & 16.3 & 110.6 & 127.7 & 59.5 & 103.1 & 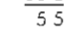 & 24.0 & 16.0 \\
\hline Mud & 6.7 & 54.0 & 125.4 & 1.8 & & & $=$ & \\
\hline \multicolumn{9}{|c|}{ Pseudodiaptomus colefaxi cop. } \\
\hline Coral & & & & & 109.7 & 40.6 & 78 & 26.0 \\
\hline Gras & 14.2 & 50.9 & 101.5 & 787 & & & $=$ & \\
\hline Mud & 5.9 & 34.7 & 1574 & 29.4 & & & $=$ & \\
\hline \multicolumn{9}{|l|}{ Acarta spp. adults } \\
\hline Coral & 246.0 & 14.9 & 13787 & 40.8 & 358.7 & 244.4 & 106.2 & 170409 \\
\hline Mud & 112.9 & 188.9 & 229.5 & 32.2 & & & $=$ & \\
\hline Peltidium spp. & & & & & & & & \\
\hline Grass & & & & & 47.4 & 4.9 & 20.9 & 30 \\
\hline Mud & 2.2 & $\underline{10.5}$ & 1.4 & $\underline{9.2}$ & 7.8 & 0.7 & 0.4 & 2.2 \\
\hline Amphipods & & & & & & & & \\
\hline Grass & & & & & 3799 & 79.4 & 119.9 & 1055 \\
\hline Mud & 4.9 & 40.8 & 39.1. & 28.2 & & & $=$ & \\
\hline Cumaceans & & & & & & & & \\
\hline Grass & 68.1 & $\underline{89.5}$ & 20.0 & 10.8 & 90.7 & 63 & 11.8 & 161 \\
\hline Mud & $\overline{0}$ & $\overline{40}$ & 3.2 & 6.9 & & & $=$ & \\
\hline Mysids & & & & & & & & \\
\hline Coral & 14.5 & 62.1 & 55.1 & 82.3 & & & $=$ & \\
\hline Grass & & & & & 28.8 & 55 & $\underline{29} 2$ & 29.4 \\
\hline Mud & 35 & 10.6 & 13.8 & $\underline{35.1}$ & & & $=$ & \\
\hline Sagitta spp & & & & & & & & \\
\hline Coral & 184.4 & 702 & 397.7. & 18.4 & 1.8 & 1.8 & 1.8 & 11.0 \\
\hline Grass & 2125 & 399.7 & 235.5 & 51.0 & 60 & 0.4 & 04 & 1.4 \\
\hline Mud & 3093 & 255.8 & 267.1 & 29.5 & & & $=$ & \\
\hline
\end{tabular}

females, were rare in re-entry traps, emerged at night, and were equally common in daytime and night-time net tows. This calanoid appears to have been distributed throughout the water column during the day with an increase in numbers near the surface at night. Perhaps this species should be considered a demersal zooplankter loosely associated with the substratum.

Six taxa comprised of small or transparent adults did not exhibit behavior typical of demersal zooplankton. These groups were rare in re-entry trap samples, were common in emergence trap samples, were taken in nearly equal numbers in daytime and night-time net tows, and displayed diumal emergence. Due to their behavior, these 6 taxa (Acartia spp. adults Parvocalanus sp., miscellaneous calanoids, miscellaneous cyclopoids, Metis holothuriae, and Sagitta spp.) could be considered holoplanktonic contaminants of emergence traps that were accidently captured during trap deployment or entered through gaps at the bases of the traps (Robichaux et al. 1981). 
In this study, cod ends were not attached to emergence traps until they were on the bottom; therefore, few individuals would have been trapped above $70 \mathrm{~cm}$ into the water column. Weighted skirts eliminated gaps at the bases of net traps, and RETs sealed well on the grass substratum. Gaps at the bases of the RETs were less than $4 \mathrm{~cm}$ on coral and mud bottoms, therefore zooplankters swept into any gaps must have been within a few centimeters of the bottom.

The capture of reasonable numbers of these 6 taxa by RETs and net emergence traps from all types of bottom was not regarded as only incidental. Populations of these taxa appear to have been distributed throughout the water column during the day, including near the bottom. Daytime distributional maxima near the bottom in shallow water have been reported for some of these taxa (Oug 1977, Stearns 1986, Ueda 1987, Jacoby $\&$ Greenwood 1988). These taxa did not fit a strict definition of demersal zooplankton because they were found in the water column during the day and they showed daytime emergence. However, their presence near the bottom may be important to future studies of zooplankton in shallow water ecosystems.

Oithona spp., miscellaneous harpacticoids, tanaids, larvaceans, and gastropods were most common in reentry traps or night tows but they exhibited diurnal emergence patterns. Gastropods obviously did not swim into re-entry or emergence traps and were considered to be contaminants due to crawling. Tanaids fit the pattern expected for demersal zooplankton except they emerged primarily into the first sampling bottle (15:00 to 16:30 h). Only low numbers emerged, and movement may have been a result of disturbance during trap deployment. Therefore, tanaids were regarded as demersal animals that did not emerge in large numbers. Miscellaneous harpacticoids typically emerged in equal numbers throughout the $21 \mathrm{~h}$ sampling period except for one instance of peak emer-
Fig. 1. Mean number of zooplankters emerging $\mathrm{m}^{-2} 90 \mathrm{~min}^{-1}(\mathrm{n}=2)$. (a) Pseudodiaptomus colefaxi males from summer, last quarter, mud; (b) $P$. colefaxi ovigerous females from summer, last quarter, mud; (c) P. colefaxi males from summer, first quarter, coral; (d) polychaetes from summer, new moon, coral; (e) $P$. colefaxi males from summer, new moon, grass; (f) $P$. colefaxi ovigerous females from summer, new moon, grass; ( $g$ ) Acartia spp. adults from summer, last quarter, coral; (h) miscellaneous harpacticoids from summer, first quarter, mud. $S S, S R$, and vertical rules: sunset and sunrise; MR: moonrise; MS: moonset; $\mathrm{H}$ : high tide; $\mathrm{L}$ : low tide. a: mean is significantly greater than unlabeled means as shown by ANOVA and S-N-K tests $(p>0.05)$

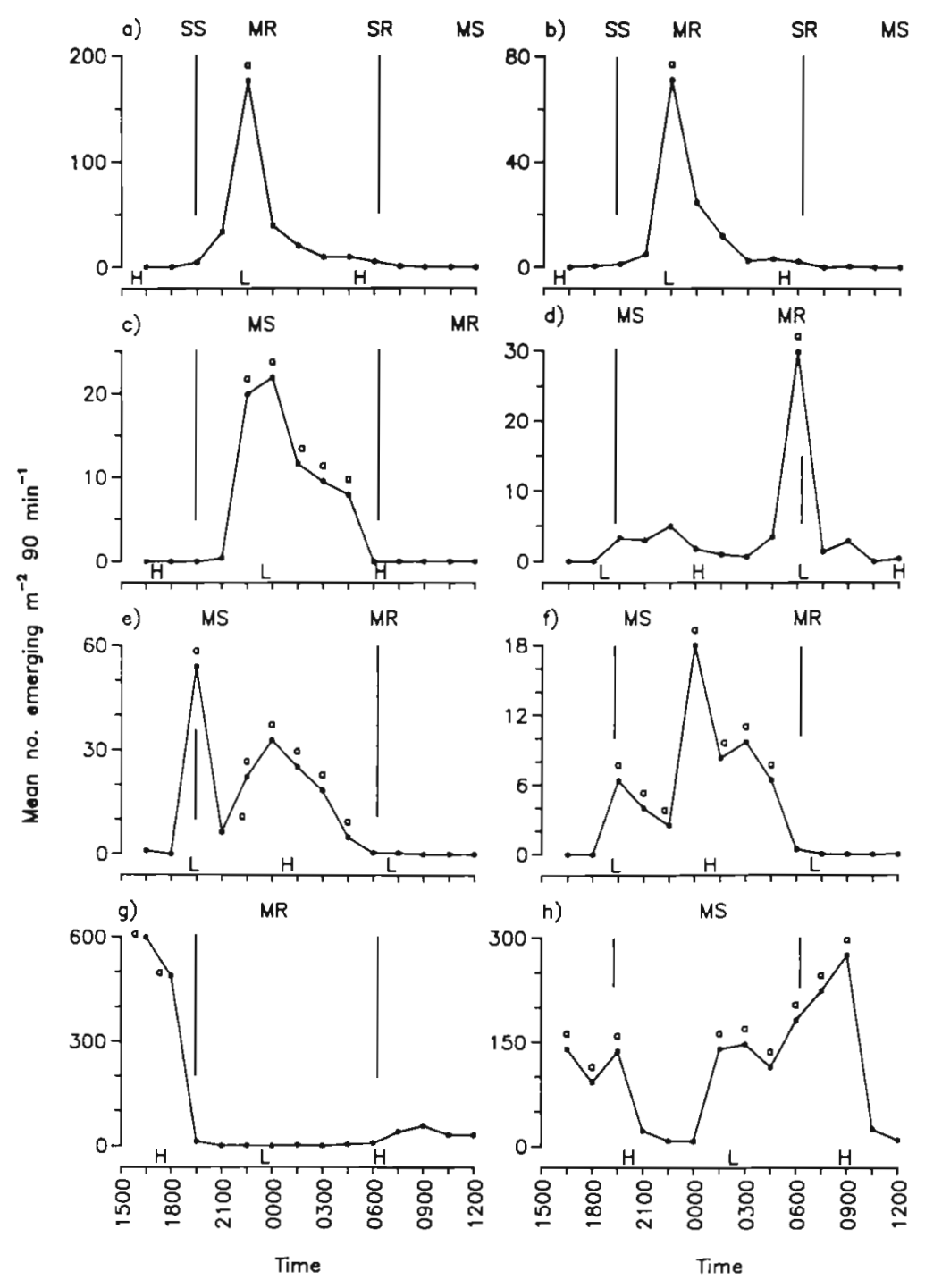


Table 9. Taxa displaying significant differences in numbers captured in RETs among substrata. Values are mean nos. $\mathrm{m}^{-2}$ night ${ }^{-1}$ $(n=2)$; means are back-transformed from $\log (c+1)$; underlined means are significantly greater within taxon, season, and lunar period (ANOVA and S-N-K tests, $p<0.05$ ). FQ: first quarter; FM: full moon; LQ: last quarter; NM: new moon; cop.. copepodites; $=$ : means are equal

\begin{tabular}{|c|c|c|c|c|c|c|}
\hline \multirow[t]{2}{*}{ Taxon/lunat period } & \multicolumn{3}{|c|}{ Summer } & \multicolumn{3}{|c|}{ Winter } \\
\hline & Coral & Grass & Mud & Coral & Grass & Mud \\
\hline \multicolumn{7}{|c|}{ Coral $>$} \\
\hline \multicolumn{7}{|c|}{$P_{\text {seudodiaptomus colefax cop. }}$} \\
\hline$F Q$ & 60.3 & 142 & 5.9 & & $=$ & \\
\hline \multicolumn{7}{|l|}{ Ridgewaya spp. } \\
\hline $\mathrm{FQ}$ & 14.6 & 2.5 & 0 & & $=$ & \\
\hline FM & 53.7 & 9.5 & 47 & & $=$ & \\
\hline LQ & 40.6 & 12.3 & 9.2 & & $=$ & \\
\hline NM & 18.0 & 104 & 1.8 & & $=$ & \\
\hline \multicolumn{7}{|c|}{ Miscellaneous calanoids } \\
\hline $\mathrm{NM}$ & & $=$ & & 97.6 & 6.4 & 5.7 \\
\hline \multicolumn{7}{|l|}{ Calanoid cop } \\
\hline NM & & $=$ & & 4190.1 & 743.7 & 1279.4 \\
\hline \multicolumn{7}{|l|}{ Oithona sp. } \\
\hline NM & & $=$ & & 1893.4 & 392.9 & 441.7 \\
\hline Larvaceans & & & & & & \\
\hline$F Q$ & & $=$ & & 70.3 & 117 & 15.6 \\
\hline LQ & & $=$ & & 23.7 & 3.6 & 5.6 \\
\hline $\mathrm{NM}$ & 181.3 & 187.5 & 340 & 10.350 .4 & 2166.5 & 1448.6 \\
\hline Polychaete larvae & & & & & & \\
\hline $\mathrm{FQ}$ & 287.0 & 58.0 & 425 & & $=$ & \\
\hline Coral, Grass > & & & & & & \\
\hline Pseudodiaptomus m & & & & & & \\
\hline $\mathrm{FQ}$ & 238.9 & 99.0 & 11.9 & & $=$ & \\
\hline FM & 73.1 & 29.2 & 7.3 & & $=$ & \\
\hline$L Q$ & 101.3 & 34.5 & 11.6 & & $=$ & \\
\hline $\mathrm{NM}$ & 32.1 & 138 & 4.2 & 19.9 & 1.0 & 10 \\
\hline Pseudodiaptomus $m$ & & & & & & \\
\hline $\mathrm{FQ}$ & 140.2 & $96 ?$ & 16.8 & & & \\
\hline FM & & $=$ & & $\underline{2.9}$ & 0 & 0.4 \\
\hline LQ & 57.9 & 32.1 & 9.5 & & $=$ & \\
\hline NM & 40,7 & 30.6 & 3.6 & 6.4 & 0 & 3.5 \\
\hline Pseudodiaptomus m & & & & & & \\
\hline$F Q$ & 554.0 & 84.1 & 51.7 & & $=$ & \\
\hline $\mathrm{FM}$ & 197,1 & 123.7. & 470 & & $=$ & \\
\hline NM & 147.1 & 106.2 & 37.8 & 10.6 & 10 & 0 \\
\hline Pseudocyclops spp. & & & & & & \\
\hline $\mathrm{FQ}$ & 5.0 & 39 & 0.7 & 7.8 & 19.5 & 04 \\
\hline FM & 32.0 & 15.4 & 2.0 & $\overline{59}$ & 6.7 & 1.4 \\
\hline LQ & 16.0 & 3.0 & 3.0 & 15.9 & 10.5 & 0.7 \\
\hline$N M$ & 13.0 & 29 & 2.2 & 15.3 & $4 . \overline{9}$ & 2.2 \\
\hline Parvocalanus sp. & & & & & & \\
\hline$F Q$ & & $=$ & & 1053.4 & 297.8 & 351.5 \\
\hline LQ & & $=$ & & 332.3 & 286.1 & 86.7 \\
\hline Miscellareous cyclop & & & & & & \\
\hline $\mathrm{FQ}$ & & $=$ & & 103.9 & 107.3 & 22.8 \\
\hline LQ & & $=$ & & 223.7 & 224.0 & 72.9 \\
\hline Amphipods & & & & & & \\
\hline FQ & 27.6 & 70.4 . & 4.9 & 87.4 & 379.9 & 104.5 \\
\hline Curnaceans & & & & & & \\
\hline$F Q$ & 11.6 & 68.1. & 0 & 12,0 & 90.7 & 13.1 \\
\hline$F M$ & 46.7 & 89.5 & 4.0 & & $=$ & \\
\hline LQ & 43.5 & 20.0. & 32 & & $=$ & \\
\hline Ostracods & & & & & & \\
\hline$F Q$ & 60.4 & 446.9 & 1167 & 46.4 & 14.5 & 10.5 \\
\hline LQ & & $=$ & & 15.5 & 7.9 & 20 \\
\hline Cirnpede laryae & & & & & & \\
\hline$F Q$ & $\underline{27.9}$ & $\underline{21.2}$ & 6.0 & & $=$ & \\
\hline NM & & $=$ & & 329.3 & 72.8 & 52.9 \\
\hline Gastropods veligers & & & & & & \\
\hline$F Q$ & 121.4 & 92.4. & 340 & & $=$ & \\
\hline NM & & $=$ & & $\underline{14859}$ & 2141 & 3716 \\
\hline Bivalve veligers & & & & & & \\
\hline$F Q$ & 3833.3 & 1210.3 & 3774 & 7.0 & 16.9 & 0.4 \\
\hline FM & 5291.4 & 1609.1 & 772.1 & & $=$ & \\
\hline La & 1550.4 & 43.49 .4 & 1293.1 & & $=$ & \\
\hline NM & & $=$ & & 4752.9 & 935.6 & 1247.2 \\
\hline Grass $>$ & & & & & & \\
\hline Parategastes sp. & & & & & & \\
\hline$F Q$ & 199.9 & 657.6 & 56.3 & 130.3 & 10207.2 & 243.5 \\
\hline$F M$ & 102.6 & 651.9 & 131.7 & 1.78 .6 & 1724.9 & 335.9 \\
\hline $\mathrm{LQ}$ & 858 & 4124 & 320 & 320.1 & 21527 & 103.5 \\
\hline $\mathrm{NM}$ & & $=$ & & 167.1 & 960.2 & $568 ?$ \\
\hline
\end{tabular}


Table 9 (continued)

\begin{tabular}{|c|c|c|c|c|c|c|}
\hline \multirow[t]{2}{*}{ Taxon/lunar period } & \multicolumn{3}{|c|}{ Summer } & \multicolumn{3}{|c|}{ Winter } \\
\hline & Coral & Grass & Mud & Coral & Grass & Mud \\
\hline \multicolumn{7}{|l|}{ Grass > } \\
\hline \multicolumn{7}{|l|}{ Peltidium sp. } \\
\hline $\mathrm{FQ}$ & & $=$ & & 2.9 & 474 & 7.8 \\
\hline $\mathrm{FM}$ & & $=$ & & 0.7 & 49 & 0.7 \\
\hline LQ & 2.9 & 10.5 & 1.4 & 1.0 & 209 & 0.4 \\
\hline \multicolumn{7}{|c|}{ Miscellaneous harpacticoids } \\
\hline$F Q$ & & $=$ & & 744.8 & 67410 & 918.7 \\
\hline LQ & & $=$ & & 785 & 2433.4 & 395.3 \\
\hline \multicolumn{7}{|l|}{ Isopods } \\
\hline EM & 0.4 & $\underline{5.3}$ & 12 & & $=$ & \\
\hline Spadella cephaloptera & & & & & & \\
\hline FM & 10 & $\$ 4.3$ & 1.4 & & $=$ & \\
\hline Grass, Mud > & & & & & & \\
\hline Pseudodiaptomus colet & & & & & & \\
\hline$F Q$ & 784 & 55.4 & 117 & 368 & 289.1 & 151.4 \\
\hline LQ & 25.0 & 311.1 & 304.2 & 7.8 & $\underline{55.9}$ & 31.8 \\
\hline$N=1$ & 46.0 & 175.4 & 210 & & $=$ & \\
\hline Pseudodiaptomus colef & & & & & & \\
\hline$F Q$ & & $=$ & & 7.9 & 1031 & 40.8 \\
\hline$F M$ & 154 & 110.6 & 54.0 & $\underline{33.2}$ & 5.5 & $\underline{26.5}$ \\
\hline LQ & 8.5 & 127.7 & 125.4 & & $=$ & \\
\hline$N M$ & 15.3 & 59.5 & 18 & & $=$ & \\
\hline Metis holothunae & & & & & & \\
\hline$F Q$ & 5.0 & 24.1 & 2.7 & 47 & 92.2 & 11.5 \\
\hline FM & 0.4 & 30.7 & 35.9 & 07 & 8.9 & 3.9 \\
\hline$L Q$ & 0.7 & 12.5 & 79 & 2.0 & 22.0 & 4.5 \\
\hline NM & 6.3 & 49.1 & 44.7 & 0 & 10.7 & 3.2 \\
\hline Carideans & & & & & & \\
\hline$F Q$ & 8.8 & 29.6 & 5.9 & & $=$ & \\
\hline $\mathrm{FM}$ & 1.0 & 8.8 & 2.5 & & $=$ & \\
\hline NM & & $=$ & & 0.4 & 0 & 35 \\
\hline Caridean/anomuran lar & & & & & & \\
\hline $\mathrm{FM}$ & 276 & 80.6 & 92.7 & 0 & 8.8 & 13. \\
\hline Tanaids & & & & & & \\
\hline $\mathrm{FQ}$ & 55 & 410 & 1.4 & 30 & 19.8 & 59 \\
\hline $\mathrm{FM}$ & 30 & 35.7 & 16.3 & 04 & 9.6 & 8.8 \\
\hline LQ & 18 & 16.1 & 25 & & $=$ & \\
\hline NM & 04 & 180 & $\underline{11.8}$ & 1.0 & 18.5 & 15.0 \\
\hline Megalopa & & & & & & \\
\hline $\mathrm{FQ}$ & 0.4 & 7.4 & 04 & 0 & 9.2 & 7.8 \\
\hline$F M$ & 0.4 & $200 \overline{5}$ & 1.0 & & $=$ & \\
\hline$L Q$ & 1.0 & 5.9 & 14 & & $=$ & \\
\hline NM & & $=$ & & 0 & 1.4 & 16.7 \\
\hline Gastropods & & & & & & \\
\hline $\mathrm{FM}$ & & $=$ & & 0 & 17.4 & 14.3 \\
\hline $\mathrm{LQ}$ & & $=$ & & 04 & 5.3 & $\overline{0}$ \\
\hline$N M$ & & $=$ & & 04 & 12.4 & 6.5 \\
\hline $\begin{array}{l}\text { Coral, Mud > } \\
\text { Acartia spp adults }\end{array}$ & & & & & & \\
\hline$F Q$ & & $=$ & & 358.7 & 65.4 & 363.3 \\
\hline FM & 14.9 & 698 & 188.9 & 244.4 & 73.2 & 238.5 \\
\hline $\mathrm{LQ}$ & 1378.7 & 221.7 & 229.5 & & & \\
\hline NM & & $=$ & & 17040.9 & 407.7 & 532.0 \\
\hline Acartua spp cop. & & & & & & \\
\hline $\mathrm{FQ}$ & & $=$ & & 335.5 & 2411 & 1265.4 \\
\hline NM & & $=$ & & 16448.9 & 8014 & 1541.2 \\
\hline Substrata $=$ & & & & & & \\
\hline Pseudodiaptomus colef & & & & & & \\
\hline$F Q$ & 24 ? & 14.5 & 2.9 & & $=$ & \\
\hline FM & & $=$ & & 38.5 & 7.1 & $\underline{28.0}$ \\
\hline $\mathrm{LQ}$ & 17.0 & 84.0 & 84.0 & & $=$ & \\
\hline $\mathrm{NM}$ & 275 & 100.0 & $\overline{24.4}$ & & $=$ & \\
\hline Stephos spp. & & & & & & \\
\hline$F Q$ & 1.8 & 11.8 & 2.2 & $\underline{39.7}$ & 36.1 & 7.5 \\
\hline$F M$ & 3.0 & 30.3 & 3.5 & 39.4 & 28.8 & 8.9 \\
\hline$L Q$ & 22 & $\frac{99}{99}$ & 75 & & $=$ & \\
\hline $\mathrm{NM}$ & 3.5 & 28.0 & $2 \overline{6.5}$ & & $=$ & \\
\hline Mysids & & & & & & \\
\hline$F Q$ & 14.5 & 42.9 & 3.5 & 3.2 & $\underline{28.8}$ & 10.0 \\
\hline FM & $62 \pi$ & $\overline{56.2}$ & 10.6 & & $=$ & \\
\hline$L Q$ & 65.1 & 38.6 & 13.8 & 6.5 & 29.2 & $\underline{26.5}$ \\
\hline NM & & $=$ & & 5.0 & 29.4 & $\underline{44.0}$ \\
\hline Zoeae & & & & & & \\
\hline $\mathrm{FQ}$ & 137.2 & 19.0 & 14.7 & & $=$ & \\
\hline$F M$ & $\underline{53.3}$ & 111.6 & 140 & 3.9 & 39.2 & 18.2 \\
\hline LM & & $=$ & & 5.0 & $\underline{15.2}$ & 4.0 \\
\hline NM & & $=$ & & 5.3 & $\overline{14.2}$ & 46.3 \\
\hline
\end{tabular}


Table 9 (continued)

\begin{tabular}{|c|c|c|c|c|c|c|}
\hline \multirow[t]{2}{*}{ Taxon/lunar period } & \multicolumn{3}{|c|}{ Summer } & \multicolumn{3}{|c|}{ Winter } \\
\hline & Coral & Grass & Mud & Coral & Grass & Mud \\
\hline \multicolumn{7}{|l|}{ Substrata $=$} \\
\hline \multicolumn{7}{|l|}{ Miscellaneous nauphii } \\
\hline$F Q$ & 270 & 200 & 50 & & & \\
\hline NM & 115.9 & 26.5 & 25.1 & 54.0 & 5.9 & 38.7 \\
\hline \multicolumn{7}{|l|}{ Saqitia spp. } \\
\hline$F M$ & 70.2 & 399.7 & 255.8 & & $=$ & \\
\hline NM & & $=$ & & $\underline{11.0}$ & 1.4 & 0.4 \\
\hline \multicolumn{7}{|l|}{ Polychaetes } \\
\hline $\mathrm{FQ}$ & & $=$ & & 2770 & 90.0 & 29.2 \\
\hline$F M$ & & $=$ & & 5.0 & 23.5 & 18.7 \\
\hline LQ & 172.2 & 55.3 & 11.4 & 189.2 & 92.2 & 7.9 \\
\hline \multicolumn{7}{|l|}{ Tunicate larvae } \\
\hline FM & $\underline{17.4}$ & 2.00 & 3.4 & 7.0 & 4.5 & 0.4 \\
\hline
\end{tabular}

gence during the day. This multispecific group probably contained some demersal taxa, as well as other holoplanktonic species (Walters \& Bell 1986). Oithona spp. and larvaceans were taken in greater numbers in night-time net tows yet they displayed diurnal emergence patterns. Larvaceans were captured largely in the first sampling bottle and their emergence may have been caused by disturbance during trap deployment. Oithona spp. were taken in equal numbers in re-entry and emergence traps suggesting that some members of this genus were very close to the bottom during the day and could be classified as demersal zooplankters. Although Oithona spp, and larvaceans typically are considered holoplankters, their daytime distribution in shallow water appears to extend very near the bottom because they have been taken in emergence traps at several locations (Porter et al. 1977, Feeley et al. 1979, Ohlhorst 1982, Jacoby \& Greenwood 1988).

Fourteen taxa in this study consisted of larval forms. Results indicated that only zoeae in the winter were more common near the surface during the day. Pseudodiaptomus colefaxi and Pseudodiaptomus mertoni copepodites, caridean/anomuran larvae, zoeae, megalopa, and bivalve veligers emerged predominantly at night. These taxa appeared to be near the bottom during the day. Gastropod veligers, which were more numerous in night tows, also may have belonged in this category if their statistically significant emergence into the first sampling bottle was due to disturbance. Later instars of Pseudodiaptomus spp. copepodites apparently exhibited a demersal habit similar to the adults of these species. Caridean/anomuran larvae, zoeae, and megalopa were large relative to most larvae, and they tended to be near the bottom during the day which could have reduced their availability to visual predators. In contrast, smaller or transparent larval forms which may have been less subject to visual predation, i.e. Acartia spp. copepodites, calanoid copepodites, cirripede larvae, and polychaete larvae, were common in daytime net tows and showed diurnal emergence or no pattern in emergence. Copepod nauplii, miscellaneous nauplii, and tunicate larvae probably should have been included in this assemblage because they exhibited diurnal emergence, but they were too small to be taken in net tows.

The taxa captured in emergence and re-entry traps in this study were similar to those previously for coral reefs (Alldredge \& King 1977, Porter et al. 1977, Randall et al. 1978, Feeley et al. 1979, Hobson \& Chess 1979, Birkeland \& Smalley 1981, McWilliam et al. 1981, Walter et al. 1981, Ohlhorst 1982, Jacoby \& Greenwood 1988), for subtidal sand flats (Alldredge \& King 1980, 1985, Youngbluth 1982a, Stretch 1985), for seagrass beds (Robichaux et al. 1981, Alldredge 1985, Bell et al. 1986, Hicks 1986, Walters \& Bell 1986), for kelp beds (Hammer \& Zimmerman 1979, Hammer 1981), and for a temperate estuary (Thomas \& Jelley 1972). Comparisons of numbers of zooplankters captured were hampered by differences in classification and methodology, but the total numbers of zooplankters captured in this study (emergence traps: 276 to 97888 individuals $\mathrm{m}^{-2}$ night ${ }^{-1}$; re-entry traps: 3137 to 103666 individuals $\mathrm{m}^{-2}$ ) generally were within reported ranges. The closest comparisons could be made to a study in the lagoon of Heron Reef, Great Barrier Reef (Jacoby \& Greenwood 1988). The number of zooplankters captured in Moreton Bay was an order of magnitude greater for most taxa. Past studies have concentrated on the importance of demersal zooplankton in the trophic webs of coral reefs, but this assemblage may be equally important in estuaries.

All types of sampling used in this study were effective, but each also appeared to have biases. Net tows at the surface tended to capture smaller forms and missed many demersal taxa, even at night. These results indicated that most animals did not move to the surface of the 1 to $4 \mathrm{~m}$ water column or some taxa avoided the net. Re-entry traps and emergence traps captured equal numbers of 19 out of 42 taxa. Eight groups, mainly larger demersal zooplankters and crawling gastropods, 
Table 10. Occurrences of statistically significant temporal emergence patterns within season/lunar period/substratum. Peaks in emergence greater than 10 zooplankters $\mathrm{m}^{-2} 90 \mathrm{~min}^{-1}$ Su: summer; Wi: Winter; FQ: first quarter; FM: full moon; LQ: last quarter; NM: new moon; Co: coral; Gr: grass; Mu: mud; -: no effect for levels of this factor; --: emergence pattern not shown by this taxon; cop: copepodites

\begin{tabular}{|c|c|c|c|c|c|}
\hline Taxon & Sunset & Nocturna! & Sunrise & Bimodal & Diurnal \\
\hline Pseudodiaptomus colefaxi to & $\begin{array}{l}\text { Su/FQ/Gr } \\
\text { Si/LQ/Gr } \\
\text { Su/LQ/Mu } \\
\text { Wi/FQ/Gr } \\
\text { Wi/FQ/Mu } \\
\text { Wi/FM/Co } \\
\text { Wi/FM/Mu } \\
\text { Wi/LQ/Gr } \\
\text { Wi/LQ/Mu }\end{array}$ & $\begin{array}{l}\mathrm{Su} / \mathrm{FQ} / \mathrm{Co} \\
\mathrm{Su} / \mathrm{FM} / \mathrm{Co}\end{array}$ & -- & $\begin{array}{l}\text { Su/FM/Mu } \\
\mathrm{Su} / \mathrm{NM} / \mathrm{Gr} \\
\mathrm{Wi} / \mathrm{Nm} / \mathrm{Mu}\end{array}$ & -- \\
\hline$P$ colefaxi ९९ & $\begin{array}{l}\text { Wi/FM/Co } \\
\text { Wi/FM/Mu } \\
\text { Wi/LQ/Mu }\end{array}$ & -- & - & $\cdots$ & -- \\
\hline P. colefaxi ovigerous $9 \%$ & $\begin{array}{l}\text { SW/LQ/Gr } \\
\text { Wi/FQ/Gr } \\
\text { Wi/FQ/Mu } \\
\text { Wi/FM/Co } \\
\text { Wi/FM/Mu } \\
\text { Wi/LQ/Cr }\end{array}$ & $\begin{array}{l}\mathrm{Su} / \mathrm{FM} / \mathrm{Gr} \\
\mathrm{Su} / \mathrm{FM} / \mathrm{Mu}\end{array}$ & -- & $\begin{array}{l}\text { Su/LQ/Mu } \\
\text { Su/NM/Gr }\end{array}$ & - \\
\hline P. colefaxi cop. & $\begin{array}{l}\text { Wi/FQ/Gr } \\
\text { Wi/FM/Co } \\
\text { Wi/FM/Mu }\end{array}$ & $\mathrm{Su} / \mathrm{FM} / \mathrm{Mu}$ & -- & $\cdots$ & - \\
\hline Pseudodiaptomus mertoni do & $\begin{array}{l}\mathrm{Su} / \mathrm{FQ} / \mathrm{Gr} \\
\mathrm{Su} / \mathrm{FM} / \mathrm{Co} \\
\mathrm{Su} / \mathrm{FM} / \mathrm{Gr}\end{array}$ & $\mathrm{Su} / \mathrm{FQ} / \mathrm{Co}$ & -- & $\cdots$ & - \\
\hline$P$ merton 99 & $\begin{array}{l}\text { Su/FQ/Gr } \\
\mathrm{Su} / \mathrm{FM} / \mathrm{Co} \\
\mathrm{Su} / \mathrm{LQ} / \mathrm{Gr}\end{array}$ & $\mathrm{Su} / \mathrm{FQ} / \mathrm{Co}$ & -- & -- & $\cdots$ \\
\hline P. mertonicop. & -- & $\begin{array}{l}\mathrm{Su} / \mathrm{FQ} / \mathrm{Co} \\
-\end{array}$ & -- & $\cdots$ & Su/FM/Gr \\
\hline Stephos spp. & Wi/QF/Gr & Wi/FM/Gr & -- & -- & -- \\
\hline Acartia sp. adults & -- & -- & - & $\cdots$ & $\mathrm{Su} / \mathrm{LQ} / \mathrm{Co}$ \\
\hline Pseudocyclops spp & $\begin{array}{l}\mathrm{Su} / \mathrm{FM} / \mathrm{Co} \\
\mathrm{W} / \mathrm{NM} / \mathrm{Co}\end{array}$ & -- & - & -- & -- \\
\hline Ridgewayia sp. & -- & $\mathrm{Su} / \mathrm{FM} / \mathrm{Co}$ & - & $\mathrm{Su} / \mathrm{L} Q \mathrm{CO}$ & -- \\
\hline Miscellaneous calanoids & -- & -- & -- & -- & $\begin{array}{l}\text { Su/FQ/Co } \\
\text { Sw/FQ/MU }\end{array}$ \\
\hline Oithona spp & -- & - & -- & - & $\mathrm{Su} / E \mathrm{QQ} / \mathrm{Mu}$ \\
\hline Miscellaneous harpacticonts & -- & - & - & - & $\mathrm{Su} / \mathrm{FQ} / \mathrm{Mu}$ \\
\hline Copepod nauplii & -- & -- & -- & -- & $\begin{array}{l}-/-/ \mathrm{Co} \\
-/ / \mathrm{Gr}\end{array}$ \\
\hline Anphipods & $\begin{array}{l}\mathrm{Su} / \mathrm{LQ} / \mathrm{Co} \\
\mathrm{Su} / \mathrm{NM} / \mathrm{Co} \\
W_{1} / \mathrm{FQ} / \mathrm{Co} \\
W_{\mathrm{i}} / \mathrm{FM} / \mathrm{Co} \\
\mathrm{W}_{1 / \mathrm{FM} / \mathrm{Mu}} \\
\mathrm{Wi}_{\mathrm{i}} / \mathrm{LQ} / \mathrm{Co}\end{array}$ & - & -- & -- & -- \\
\hline Cumaceans & $\begin{array}{l}\mathrm{Su} / \mathrm{FQ} / \mathrm{Gr} \\
\mathrm{Su} / \mathrm{FM} / \mathrm{CO} \\
\mathrm{Su} / \mathrm{FM} / \mathrm{Gr} \\
\mathrm{Su} / \mathrm{QQ} / \mathrm{Co} \\
\mathrm{Su} / \mathrm{LQ} / \mathrm{Gr} \\
\mathrm{Wi} / \mathrm{FQ} / \mathrm{Gr} \\
\mathrm{Wi} / \mathrm{NM} / \mathrm{Gr}\end{array}$ & -- & - & - & -- \\
\hline Mysids & $\begin{array}{l}\text { Su/FM/Co } \\
\text { Su/FM/Gr } \\
\text { Su/LQ/Co } \\
\text { Su/LQ/Gr } \\
\text { Su/NM/Co } \\
\text { Su/NM/Mu } \\
\text { Wi/LQ/Mu } \\
\text { Wi/NM/Gr }\end{array}$ & -- & $\cdots$ & -- & -- \\
\hline Ostracods & Wi/FQ/- & -- & -- & -- & -- \\
\hline Candean/anomuran larvae & -- & $\begin{array}{l}\mathrm{Su} / \mathrm{FM} / \mathrm{Co} \\
\mathrm{Su} / \mathrm{FM} / \mathrm{Mu} \\
\mathrm{Su} / \mathrm{NM} / \mathrm{Gr} \\
\mathrm{Su} / \mathrm{NM} / \mathrm{Mu}\end{array}$ & -- & $\cdots$ & -- \\
\hline Zoeae & -- & $-/ \mathrm{FQ} / \mathrm{Co}$ & - & -- & -- \\
\hline Megalopa & $\mathrm{Su} / \mathrm{FM} / \mathrm{Gr}$ & - & -- & $\mathrm{Wi} / \mathrm{NM} / \mathrm{Mu}$ & -- \\
\hline
\end{tabular}


Table 10 (continued)

\begin{tabular}{|c|c|c|c|c|c|}
\hline Taxon & Sunset & Nocturnal & Sunnse & Bimiódal & Diuma! \\
\hline Sagitta spp. & $\cdots$ & $\cdots$ & - & - & $\begin{array}{l}\mathrm{Su} / \mathrm{FQ} / \mathrm{CO} \\
\mathrm{Su} / \mathrm{LQ} / \mathrm{Co}\end{array}$ \\
\hline Larvaceans & -- & $\ldots$ & -. & -- & $\mathrm{Su} / \mathrm{LQ} / \mathrm{CO}$ \\
\hline Polychaetes & $\begin{array}{l}\text { Su/FQ/Gr } \\
\text { Wi/FQ/Co } \\
\text { Wi/FQ/Gr } \\
\text { Wi/FQ/Mu }\end{array}$ & $\begin{array}{l}\text { Su/NM/Gr } \\
\text { Wi/LQ/Col }\end{array}$ & $\mathrm{Su} / \mathrm{NM} / \mathrm{CO}$ & $\begin{array}{l}\mathrm{Su} / \mathrm{LQ} / \mathrm{CG} \\
\text { Wi/LQ/GT }\end{array}$ & -- \\
\hline Polychaete larvae & -. & $\cdots$ & -. & - & $\mathrm{W}_{\mathrm{i} /}-1-1$ \\
\hline Gastropod veligers & -. & -- & -. & -- & All \\
\hline Bivalve veligers & -- & $\mathrm{Su} / \mathrm{FM} / \mathrm{Co}$ & -- & -- & -- \\
\hline
\end{tabular}

Table 11. Distribution of significant night-time emergence patterns with peaks of more than 10 individuals $\mathrm{m}^{-2} 90 \mathrm{~min}^{-1}$ among levels of factors in ANOVAs

\begin{tabular}{|llc|}
\hline Factor & Level & $\begin{array}{c}\text { No. of signiticant } \\
\text { night-time emergence } \\
\text { patterns }\end{array}$ \\
\hline Season & Summet & 48 \\
Lunar period & Winter & 36 \\
& First quarter & 22 \\
& Full mon & 29 \\
Substratum & Last quarter & 20 \\
& New moon & 14 \\
& Coral & 32 \\
& Grass & 31 \\
& Mud & 21 \\
\hline
\end{tabular}

were more common in re-entry trap samples. These 8 taxa (i.e. Pseudodiaptomus colefaxi copepodites, Peltidium spp., amphipods, cumaceans, ostracods, polychaetes, carideans, and gastropods) were nearer the bottom during the day than the 15 groups caught in greater numbers in emergence traps. These 15 taxa, which apparently were near the bottom but did not enter defaunated sediment, included 5 groups of small demersal zooplankton (Stephos spp., Pseudocyclops spp., Ridgewayia sp.; Parategastes sp. and Spadella cephaloptera), 3 non-demersal adult taxa (Parvocalanus sp., Metis holothuriae, and larvaceans), and 7 larval taxa (miscellaneous nauplii, zoeae, caridean/ anomuran larvae, megalopa gastropod veligers, bivalve veligers, and tunicate larvae). All 43 taxa were caught in RETs and had to move at least $70 \mathrm{~cm}$ into the water column to be trapped. These results contradict hypotheses that re-entry traps consistently yield an order of magnitude more zooplankters than emergence traps (Alldredge \& King 1980) and that smaller zooplankters tend to remain nearer the bottom (Alldredge $\&$ King 1985). All of these conclusions agree with observations from a study at Heron Reef made by Jacoby \& Greenwood (1988)

Re-entry traps did not show a consistent increase in numbers of colonizers over a 3-night period. This agrees with the findings of Bell \& Devlin (1983), Chandler \& Fleeger (1983), and Jacoby \& Greenwood (1988) but differs from results reported by Stretch (1985) and Virnstein \& Curran (1986). Colonization may have decreased after one night or immigration may have been balanced by emigration. Densitydependent movement which should lead to stable levels of colonizers has been observed in amphipods and meiofauna (Ambrose 1986, Service \& Bell 1987).

Variation in zooplankton numbers attributed to seasons was more pronounced in Moreton Bay than in the lagoon of Heron Reef (Jacoby \& Greenwood 1988). In Moreton Bay, 25 out of 32 taxa of adults were taken in greater numbers in the summer in contrast to 13 out of 23 taxa at Heron Reef. At both locations, most larval taxa were captured in greater numbers in the summer (Moreton Bay: 10 out of 14 taxa; Heron Reef: 8 out of 10 taxa) indicating a summer peak in reproductive activity as has been reported elsewhere (Sale et al. 1976, 1978, McWilliam et al. 1981). Moreton Bay was expected to have stronger seasonality due to greater changes in abiotic factors, e.g. temperature, salinity, and daylength.

Lunar effects on emergence were less clear in Moreton Bay than at Heron Island (Jacoby \& Greenwood 1988). Significant temporal peaks in emergence were distributed more evenly among lunar periods for taxa from Moreton Bay. At Heron Reef, most demersal taxa (16 out of 19) emerged in greater numbers during a lunar period with part of the night moonless, but this trend was less apparent in Moreton Bay (10 out of 17 taxa). Most of the demersal taxa emerging in large numbers during the full moon in Moreton Bay, e.g., Pseudodiaptomus colefaxi, Pseudocyclops spp., and Ridgewayia spp., were smaller than the other demersal groups. Unexpectedly, amphipods and cumaceans, relatively large animals, displayed approximately equal emergence in all lunar periods at both locations. At both places, ca $40 \%$ of the non-demersal adult taxa and $80 \%$ of the larval taxa emerged in large numbers during the full moon.

Previous work indicated variable effects of lunar 
period on emergence (Alldredge \& King 1980, 1985, Ohlhorst 1982). Tidal flux may have altered emergence behavior (Palmer \& Brandt 1981, Eckman 1983, Hicks 1986, Palmer 1986), but some zooplankters emerged during all states of the tide in Moreton Bay, at Heron Reef (Jacoby \& Greenwood 1988), and at other sites (Grindley 1972, Pillai \& Pillai 1973). Turbidity in Moreton Bay generally was high and may have reduced the effect of moonlight on emergence much as darkened emergence traps did in a study by Alldredge \& King (1980). In spite of these complications, many demersal taxa emerged in greater numbers during lunar periods in which part of the night was moonless. Visual predation in the water column should be reduced during moonless portions of the night. Larval forms and small or transparent adult zooplankters emerged mainly during full and new moon periods or showed no effect of lunar period on emergence. These taxa may not be less subject to visual predation because of their size and lack of coloration, but they may accrue increased dispersal on spring tides.

Most taxa in this study $(95 \%)$ emerged in greatest numbers from coral or grass, the 2 structurally complex substrata. This trend is corroborated by results from a variety of other studies indicating that structure is often correlated with enhanced densities of animals (Alldredge \& King 1977, Porter et al. 1977. Coull et al. 1979, Feeley et al. 1979, Stoner 1980, McWilliam et al. 1981, Alldredge 1985, Stoner \& Lewis 1985, Hicks 1986 , Palmer 1986, Jacoby \& Greenwood 1988). The substrata in this study were not limited exclusively to one type of bottom, therefore, taxa from coral may have been in the surrounding rubble and taxa from grass may have been in the underlying mud. The presence of any structure above the bottom has been observed to lead to greater numbers of animals, possibly due to increased food, decreased water flow, protection from predators, or behavioral choice (Hicks 1977, Nelson 1979, Ravenel \& Thistle 1981, Stoner 1982, Coull \& Wells 1983, Edgar 1983, Thistle et al. 1984, Leber 1985, Kern \& Taghon 1986, Palmer 1986, DeWitt 1987).

By definition, daylight has a major effect on the behavior of demersal zooplankton (Jansson \& Källander 1968, Alldredge \& King 1980, Tranter et al. 1981, Walter et al. 1981, Ohlhorst 1982, Youngbluth 1982b. Renon et al. 1985, Bell et al. 1986, Walters \& Bell 1986, Jacoby \& Greenwood 1988). In this and other studies, emergence predominately occurred at night. Temporal patterns in emergence exhibited by some taxa were limited to restricted periods of time and varied among different sets of samples (Jacoby \& Greenwood 1988, this study). Emergence during restricted periods of the night will limit exposure to predation, but it also increases the likelihood of contacting a mate. Copepods, amphipods, cumaceans, and other taxa may rely on this behavior to increase the relative concentrations of conspecific individuals and sexual pheromones in the water column (Katona 1973, Griffiths \& Frost 1976, Lyes 1979, Jacoby \& Youngbluth 1983). The correspondence observed between diel emergence patterns of both sexes in the same species during different seasons and lunar periods supports this hypothesis (Jacoby \& Greenwood 1988, this study).

Emergence traps and re-entry traps rely on behavior of zooplankters for an assessment of abundance. Both types of traps appear to underestimate the total densities of zooplankters, but they have been reported to accurately reflect relative differences in abundance (Alldredge \& King 1980, Stretch 1985). Results of this study and that by Jacoby \& Greenwood (1988) indicate that conclusions regarding zooplankton abundance based on catches in re-entry and emergence traps must include an appreciation of behavioral variability.

Seasonality in emergence probably was due primarily to changes in abundance because increases in captures within taxa were consistent among lunar periods and substrata. In addition, these differences were more pronounced in the harsher environment of Moreton Bay. A seasonal peak in mating may have added a behavioral affect to the seasonality of emergence.

Substratum-related variations in emergence were consistent through all seasons and lunar periods and were seen between samples taken on the same night. These results indicated that these variations were due to stable differences in abundance, not substratumrelated behavioral effects on emergence. A behavioral choice of substratum may have contributed to the observed distributions.

In contrast, diel and lunar variations in catch rates were probably a result of behavioral changes, because lunar trends were not dependent on the order in which the moon phases were sampled and these effects were less obvious in Moreton Bay where turbidity reduced penetration of moonlight into the water column. In addition, samples from consecutive lunar periods typically were spaced over a 1 mo interval which should have reduced the effect of changes in abundance. Variations in abundance of the magnitude required to generate the observed diel patterns in emergence are unlikely in $1 \mathrm{~d}$. These patterns probably result from behavioral changes and appear to have been a response to illumination.

Results of work in Moreton Bay and at Heron Reef (Jacoby \& Greenwood 1988) suggest that it may be necessary to view demersal zooplankton as an assemblage composed of at least 2 types of animals. One group would include animals that primarily are benthic but emerge into the water column on a regular basis. A second category would include relatives of holoplankters that move to the bottom during the daytime. 
Benthic, demersal taxa (e.g. Peltidium spp., Parategastes sp, amphipods, carideans, cumaceans, isopods, ostracods, tanaids, polychaetes, and Spadella cephaloptera) probably feed while in or on the bottom. These groups, for example amphipods and cumaceans, would be very near the bottom during the day as shown by a tendency to be captured in re-entry traps (Jacoby \& Greenwood 1988, this study). Emergence would not vary significantly among lunar periods (Alldredge \& King 1980, 1985, Ohlhorst 1982, Jacoby \& Greenwood 1988, this study) because only small percentages of the populations of these animals would emerge to mate or disperse (Jones 1963, Mills 1967, Anger \& Valentin 1976, Edgar 1983, Ambrose 1986). These taxa may not rise very far into the water column and may escape net tows at the surface (Jacoby \& Greenwood 1988, this study). These benthic, demersal taxa often are subject to heavy predation by fishes feeding on the bottom during the day (Alheit 1981, Choat 1982), therefore, nocturnal, visual predators may not exert a significant selective force even if aided by moonlight. Predation may not be as strong a selective force as the need to find a mate or disperse.

The planktonic category of demersal zooplankton would include calanoid copepods (Pseudodiaptomus, Stephos, Pseudocyclops, and Ridgewayia) and mysids. Animals in these taxa probably feed (Hart 1977), mate, and disperse in the water column like their holoplanktonic relatives. A greater percentage of the populations of these zooplankters would be expected to migrate each night. These groups may move closer to the surface at night and may swarm near but not settle on or in the substratum during the day (Emery 1968, Hamner \& Carleton 1979). These behaviors would lead to their capture in night-time net tows and in daytime net tows that sampled near the bottom and to their rarity in reentry traps (Greenwood 1977, Jacoby \& Greenwood 1988 , this study). Visual predation on these taxa may be reduced if they remain near the substratum during sunlit or moonlit periods (Zaret \& Suffern 1976, Robertson \& Howard 1978, Fancett \& Kimmerer 1985). Studies of these taxa should provide clearer answers to questions concerning visual predation and the evolution of the demersal habit.

Acknowledgements. RETs, which were critical to this project, were provided by Dr. M. J. Youngbluth and the Harbor Branch Oceanographic Institution, Inc. of Fort Pierce, Florida. Invaluable support throughout this study came from the faculties and staffs of the Department of Zoology and the Dunwich Field Station, University of Queensland. Statustical analyses and production of this manuscript were carried out at the Leigh Marine Laboratory, Unıversity of Auckland. Special thanks go to D. Gaughan, M. Tucker, P. O'Connor, and K. Watson for their capable assistance with field and laboratory work. Financial support was provided by the American Philosophical Society and the Queen's Fellowship and Marine Allocations Advisory Committee (to C.A.J.), as well as the Australian Research Grants Committee (to J.G.G. \& C.A.J.)

\section{LITERATURE CITED}

Alheit, J. (1981). Feeding interactions between coral reef fishes and the zoobenthos. In: Gomez, E. D. et al. (eds.) Proc. 4 th Int. Symp. Coral Reefs 2. Marine Sciences Center, University of the Philippines, Quezon City, p. 545-552

Alheit, J, Scheibel, W. (1982). Benthic harpacticoids as a food source for fish. Mar. Biol. 70: 141-147

Alldredge, A. L. (1985). Abundance of demersal zooplankton on tropical seagrass meadows. Res. Rep. natl geogr Soc., Wash. 19: 95-101

Alldredge, A. L., King, J. M. (1977). Distribution, abundance, and substrate preference of demersal reef zooplankton at Lizard Island Lagoon, Great Barrier Reef. Mar Biol. 41. 317-333

Alldredge, A. L., King, J. M. (1980). Effects of moonlight on the vertical migration patterns of demersal zooplankton. J. exp. mar. Biol. Ecol. 44: 133-156

Alldredge, A. L., King, J. M. (1985). The distance demersal zooplankton migrate above the benthos: implications for predation. Mar Biol. 84: 253-260

Ambrose, W. G., Jr (1986). Experimental analysis of density dependent emigration of the amphipod Rhepoxynius abronis. Mar. Behav. Physiol. 12: 209-216

Anger, K. C., Valentin, C. (1976). In situ studies on the diumal activity pattern of Diastylis rathkei (Cumacea, Crustacea) and its importance for the 'hyperbenthos'. Helgoländer wiss. Meeresunters. 28: 138-144

Bell, S. S., Devlin, D. J. (1983). Short-term recolonization of sediment and epibenthic habitats in Tampa Bay, Florida. Bull. mar. Sci. 38: 102-108

Bell, S. S., Kern, J. C., Walters, K. (1986). Sampling for meifaunal taxa in seagrass systems: lessons from studies of a subtropical Florida estuary, USA. In: Thompson, M., Sarojini, R., Nagabhushanam, R. (eds.) Indian Ocean - biology of benthic organisms. A. A. Balkema, Rotterdam, p. $239-245$

Birkeland, C., Smalley, T L. (1981). Comparisons of demersal plankton from comparable substrata from a high island and an atoll. In: Gomez, E. D. et al. (eds.) Proc. 4th Int. Symp. Coral Reefs 1 Marine Sciences Center, University of the Philippines, Quezon City, p. 437-442

Chandler, G. T., Fleeger, J. W. (1983). Meiofaunal colonization of azoic estuarine sediment in Louisiana: mechanisms of dispersal. J. exp. mar. Biol. Ecol. 69: 175-188

Choat, J. H. (1982). Fish feeding and the structure of benthic communities in temperate waters. Ann. Rev. Ecol. Syst. 13: $423-429$

Coull, B. C., Bell, S. S., Savory, A. M., Dudley, B. W. (1979). Zonation of meiobenthic copepods in a southeastern United States salt marsh. Estuar, coast. Mar. Sci. 9 $181-188$

Coull, B. C. Wells, J. B. J. (1983). Refuges from fish predation: experiments with phytal meiofauna from the New Zealand rocky intertidal. Ecology 64: 1599-1609

de Morais, L. T., Bodiou, J. Y (1984). Predation on meiofauna by juvenile fish in a western Mediterranean flatfish nursery ground. Mar Biol. 82: 209-215

DeWitt, T H. (1987). Microhabit selection and colonization rates of a benthic amphipod Mar Ecol. Prog. Ser. 36: $237-250$

Eckman, J. E. (1983). Hydrodynamic processes affecting benthic recruitment. Limnol. Oceanogr 28: 241-257

Edgar, G. J. (1983). The ecology of south-east Tasmanian phytal communities. IV Factors affecting the distribution of ampithold amphipods among algae. J. exp. mar. Biol. Ecol. 70: 205-225 
Emery, A. R. (1968). Preliminary observations on coral reef plankton. Limnol. Oceanogr. 13: 293-303

Fancett, M. S., Kimmerer, J. W. (1985). Vertical migration of the demersal copepod Pseudodiaptomus as a means of predator avoidance. J. exp. Mar Biol. Ecol. 88: 31-43

Feeley, M. T., Filer, D. L., Schwartz, L. (1979). Zooplankton emerging from a Philippine coral reef: a preliminary study. J. Asian Ecol. 1: 51-56

Greenwood, J. G. (1977). Calanoid copepods of Moreton Bay (Queensland) II. Families Calocalanidae to Centropagidae. Proc. R. Soc. Queensl. 88: 49-67

Griffiths, A. M., Frost, B. W (1976). Chemical communication in the marine planktonic copepods Calanus pacificus and Pseudocalanus sp. Crustaceana 30: 1-8

Grindley, J. R. (1972). The vertical migration behaviour of estuarine plankton. Zool. Afr. 7: 13-20

Hammer, R. M. (1981). Day-night differences in the emergence of demersal zooplankton from a sand substrate in a kelp forest. Mar. Biol. 62: 275-280

Hammer, R. M., Zimmerman, R. C. (1979). Species of demersal zooplankton inhabiting a kelp forest ecosystem off Santa Catalina Island, California. Bull. Sth. Calif, Acad. Sci. 78: 199-206

Hamner, W.M., Carleton, J. H. (1979). Copepod swarms: attributes and role in coral reef ecosystems. Limnol. Oceanogr. 24: 1-4

Hart, R. C. (1977). Feeding rhythmicity in a migratory copepod (Pseudodiaptomus hessei [Mrázek]). Freshwater Biol. 7· 1-8

Hicks, G. R. F. (1977). Observations on substrate preferences of marine phytal harpacticoids (Copepoda). Hydrobiologia 56: $7-9$

Hicks, G. R. F. (1986). Distribution and behaviour of meiofaunal copepods inside and outside seagrass beds. Mar. Ecol. Prog. Ser, 31 159-170

Hobson, E. S., Chess, J. R. (1976). Trophic interactions among fishes and zooplankters near shore at Santa Catalina Island, California. Fish. Bull. U. S. 74:567-598

Hobson, E. S. Chess, J. R. (1978). Trophic relationships among fishes and plankton in the lagoon at Enewetak Atoll, Marshall Islands. Fish. Bull. U. S. 76: 133-153

Hobson, E. S., Chess, J. R. (1979). Zooplankton that emerge from the lagoon floor at night at Kure and Midway Atolls, Hawaii. Fish. Bull. U. S. 77-275-280

Jacoby, C. A., Greenwood, J. G. (1988). Spatial, temporal, and behavioral patterns in emergence of zooplankton in the lagoon of Heron Reef, Great Barrier Reef, Australia. Mar. Biol. 97: 309-328

Jacoby, C. A., Youngbluth, M. J. (1983). Mating behavior in three species of Pseudodiaptomus (Copepoda: Calanoida). Mar. Biol. 76: 77-86

Jansson, B. O., Källander, C. (1968). On the diurnal activity of some littoral peracarid crustaceans in the Baltic Sea. J. exp. mar Biol. Ecol. 2: 24-36

Johnson, M. W. (1949). Zooplankton as an index of water exchange between Bikini Lagoon and the open sea. Trans. Am. geophys. Un. 30: 238-244

Jones, N. S. (1963). The marine fauna of New Zealand: crustaceans of the order Cumacea. Bull. N. Z. Dep. scient. ind. Res. 152: 1-81

Katona, S. K. (1973). Evidence for sex pheromones in planktonic copepods. Limnol. Oceanogr 18: 574-583

Kern, J. C., Taghon, G. L. (1986). Can passive recruitment explain harpacticoid copepod distributions in relation to epibenthic structure? J. exp. mar. Biol. Ecol. 101: 1-23

Leber, K. M. (1985). The influence of predatory decapods, refuge, and microhabitat selection on seagrass communities. Ecology 66: 1951-1964
Lyes, M. C. (1979). The reproductive behaviour of Gammarus duebeni (Lilljeborg), and the inhibitory effect of a surface active agent. Mar Behav. Physiol. 6: 47-55

McHugh, J. L. (1967). Estuarine nekton. In. Lauff, G. H. (ed.) Estuaries. Am. Ass. Adv. Sci., Washington, D. C., p $581-620$

McWilliam, P. S., Sale, P. F., Anderson, D. T (1981). Seasonal changes in resident zooplankton sampled by emergence traps in One Tree Lagoon, Great Barrier Reef. J. exp. mar. Biol. Ecol. 52: 185-203

Mills, E. L. (1967). The biology of an Ampeliscid amphipod crustacean sibling species pair J. Fish. Res. Bd Can. 24 305-355

Nelson, W. G. (1979). Experimental studies of selective predation on amphipods: consequences for amphipod distribution and abundance. J. exp. mar. Biol. Ecol. 38: 225-245

Newell, B. S. (1971). The hydrological environment of Moreton Bay, Queensland, 1967-68. Aust. CSIRO Div. Fish. Oceanogr. Tech. Pap. 30:1-35

Ohlhorst, S. L. (1982). Diel migration patterns of demersal reef zooplankton. J. exp. mar. Biol. Ecol. 60: 1-15

Oug, E. (1977). Faunal distribution close to the sediment of a shallow marine environment. Sarsia 63: 115-121

Palmer, M. A. (1986). Hydrodynamics and structure: interactive effects on meiofauna dispersal. J. exp. mar. Biol. Ecol. 104: 53-68

Palmer, M. A., Brandt, R. R. (1981). Tidal variation in sediment densities of marine benthic copepods. Mar. Ecol. Prog. Ser. 4: $207-212$

Pillai, P. P., Pillai, M. A. (1973). Tidal influence on the diel variations of zooplankton with special reference to copepods in the Cochin Backwater. J. mar. biol Ass. India 15: $411-417$

Porter, J. W (1974). Zooplankton feeding by the Caribbean reef-building coral Montastrea cavernosa. In: Cameron, A. M. et al. (eds.) Proc. 2nd Int. Symp. Coral Reefs 1. The Great Barrier Reef Committee, Brisbane, p. 111-125

Porter, J. W., Porter, K. G. (1977). Quantitative sampling of demersal plankton migrating from different coral reef substrates. Limnol. Oceanogr. 22: 553-556

Porter, J. W., Porter, K. G., Catalan, Z. B. (1977). Quantitative sampling of Indo-Pacific demersal reef zooplankton. In: Taylor, D. L. (ed.) Proc. 3rd Int. Symp. Coral Reefs 1. School of Marine and Atmospheric Sciences, University of Miami, Miami, p. 105-112

Randall, R. H., Birkeland, C., Amesbury, S. S., Lassuy, D., Eads, J. R. (1978). Marine survey of a proposed resort site at Arakabesan Island, Palau. Tech. Rep. Univ. Guam Mar. Lab. 44: 1-66

Ravenel, W. S., Thistle, D. (1981). The effect of sediment characteristics on the distribution of two subtidal harpacticoid species. J. exp. mar Biol. Ecol. 50: 289-301

Renon, J. P., Dudemaine, M., Drouet, J. (1985). Une piège à émergence à collecteurs miltiples pour l'étude des migrations planctoniques verticales en milieu corallien. J. Plankton Res. 7: 19-34

Robertson, A. I., Howard, R. K. (1978). Diel trophic interactions between vertically-migrating zooplankton and their fish predators in an eelgrass community. Mar. Biol. 48: 207-213

Robichaux, D. M., Cohen, A. C., Reaka, M. L., Allen, D. (1981). Experiments with zooplankton on coral reefs, or, will the real demersal zooplankton please come up? P.S.Z.N.I. Mar. Ecol. 2: 77-94

Sale, P. F., McWilliam, P. S., Anderson, D. T (1976). Composition of the near-reef zooplankton at Heron Reef, Great Barrier Reef. Mar. Biol. 34: 59-66

Sale, P.F., McWilliam, P. S., Anderson, D. T. (1978). Faunal 
relationships among the near-reef zooplankton at three locations on Heron Reef, Great Barrier Reef, and seasonal changes in this fauna. Mar. Biol. 49: 133-145

SAS Institute Inc. (1985). SAS/STAT guide for personal computers, 6th edn. SAS Inst. Inc., Cary, North Carolina

Service, S. K., Bell, S. S. (1987). Density-influenced active dispersal of harpacticoid copepods. J. exp. mar. Biol. Ecol. 114: $49-62$

Stearns, D. E. (1986). Copepod grazing behavior in simulated natural light and its relation to nocturnal feeding. Mar Ecol. Prog. Ser. 30: 65-76

Stoner, A. W. (1980). The role of seagrass biomass in the organization of benthic macrofaunal assemblages. Bull. mar. Sci. 30: 537-551

Stoner, A. W. (1982). The influence of benthic macrophytes on the foraging behavior of pinfish, Lagodon rhomboides (Linnaeus). J. exp. mar. Biol. Ecol. 58: 271-284

Stoner, A. W., Lewis, F. G. III (1985). The influence of quantitative and qualitative aspects of habitat complexity in tropical sea-grass meadows. J. exp. mar. Biol. Ecol. 94: $19-40$

Stretch, J. J. (1985). Quantitative sampling of demersal zooplankton: re-entry and airlift dredge comparisons. J. exp. mar Biol. Ecol. 91: 125-136

Thistle, D., Reidenauer, J. A., Findlay, R. H. Waldo, R. (1984). An experimental investigation of enhanced harpacticoid (Copepoda) abundances around isolated seagrass shoots. Oecologia (Berl.) 63: 295-299

Thomas, M. L. H., Jelley, E. (1972). Benthos trapped leaving the bottom in Bideford River, Prince Edward Island. J. Fish. Res. Bd Can. 29: 1234-1237

Tranter, D. J., Bulleid, N. C., Campbell, R., Higgins, H. W.,
Rowe, F., Tranter, H. A., Smith, D. F. (1981). Nocturnal movements of phototactic zooplankton in shallow waters. Mar Biol. 61 317-326

Ueda, H. (1987). Small-scale ontogenetic and diel vertical distributions of neritic copepods in Maizuru Bay, Japan. Mar Ecol. Prog. Ser. 35: 65-73

Underwood, A. J. (1981). Techniques of analysis of variance in experimental marine biology and ecology. Oceanogr. mar. Biol. A. Rev. 19: 513-605

Virnstein, R. W., Curran, M. C. (1986). Colonization of artificial seagrass versus time and distance from source. Mar Ecol. Prog. Ser. 29: 279-288

Walter, T. C., Pasamonte, J. N., Talaue, L. (1981). A preliminary quantitative study on emergence of reef associated zooplankton from a Philippine coral reef. In: Gomez, E. D. et al. (eds.) Proc. 4th Int. Symp. Coral Reefs 1. Marine Sciences Center, University of the Philippines, Quezon City, p. 443-451

Walters, K. Bell, S. S. (1986). Diel patterns of active vertical migration in seagrass meiofauna. Mar. Ecol. Prog. Ser. 34 95-103

Youngbluth, M. J. (1982a). Sampling demersal zooplankton: a comparison of field collections using three different emergence traps. J. exp. Mar. Biol. Ecol. 61: 111-124

Youngbluth, M. J. (1982b). Demersal zooplankton: diel emergence patterns from subtropical seagrass habitats. EOS (Trans. Am. geophys. Un.) 63: 964

Zar, J. H. (1974). Biostatistical analysis. Prentice-Hall, New Jersey

Zaret, T. M., Suffern, J. S. (1976). Vertical migration in zooplankton as a predator avoidance mechanism. Limnol. Oceanogr. 21: 804-813

This article was submitted to the editor; it was accepted for printing on October 19,1988 